\title{
Spin structures on 3-manifolds via arbitrary triangulations
}

\author{
RICCARDO BENEDETTI \\ CARlo Petronio
}

\begin{abstract}
Let $M$ be an oriented compact 3-manifold and let $\mathcal{T}$ be a (loose) triangulation of $M$ with ideal vertices at the components of $\partial M$ and possibly internal vertices. We show that any spin structure $s$ on $M$ can be encoded by extra combinatorial structures on $\mathcal{T}$. We then analyze how to change these extra structures on $\mathcal{T}$, and $\mathcal{T}$ itself, without changing $s$, thereby getting a combinatorial realization, in the usual "objects/moves" sense, of the set of all pairs $(M, s)$. Our moves have a local nature, except one, that has a global flavour but is explicitly described anyway. We also provide an alternative approach where the global move is replaced by simultaneous local ones.
\end{abstract}

57R15; 57N10, 57M20

Combinatorial presentations of 3-dimensional topological categories, such as the description of closed oriented 3-manifolds via surgery on framed links in $S^{3}$, and many more, are among the main themes of geometric topology, and in particular have proved crucial for the theory of quantum invariants, initiated by Reshetikhin and Turaev in [18] and Turaev and Viro in [20].

A combinatorial presentation of the set of all pairs $(M, s)$, with $M$ a closed oriented 3manifold and $s$ a spin structure on $M$, was already contained in [6] by the authors. This presentation was realized by selecting the triangulations of $M$ having only one vertex and supporting a $\Delta$-complex structure (see Hatcher [10]), also called a branching. (Here triangulations are meant in a loose sense, namely the faces of each dimension are only required to have embedded interior, not embedded closure; and a loose triangulation supports a $\Delta$-complex structure if its edges can be oriented so that on each abstract tetrahedron the orientations are induced by an ordering of the vertices.) The viewpoint adopted in [6] was actually that of special spines, equivalent to that of triangulations via duality (see Matveev [16] and below). For the special spine dual to a triangulation, a branching is precisely a structure of oriented branched surface (see Williams [21]), and this structure was used in [6] to define a trivialization of the tangent bundle of $M$ along the 1 -skeleton of the spine, whence a spin structure on $M$, using constructions already proposed by Ishii [11] and Christy [9]. 
The construction just described easily extends to pairs $(M, s)$ with $M$ a compact oriented 3-manifold with nonempty boundary and $s$ a spin structure on $M$, by using branchable triangulations of $M$ with ideal vertices at the components of $\partial M$, and possibly internal vertices. Recall that a triangulation of $M$ with ideal vertices is a triangulation of the space obtained from $M$ by collapsing each component of $\partial M$ to a point, so that each collapsed component of $\partial M$ is a vertex, which is called the ideal vertex associated to the component. A triangulation of $M$ is ideal if it has ideal vertices only, and in this case the interior of $M$ is the triangulation space minus the ideal vertices. However, this approach suffers from the drawback that not all triangulations of $M$ are branchable: for instance, the canonical triangulation by two regular hyperbolic ideal tetrahedra of the hyperbolic one-cusped manifold called the "figure-eight-knot-sister" is not branchable. On the one hand, one easily sees that any triangulation of $M$ has branchable subdivisions (for example, take a regular subdivision and define a branching by choosing a total ordering of the vertices). On the other hand, in many circumstances one is interested in sticking to a given triangulation of $M$, or to consider the class of all vertex-efficient triangulations of $M$ (namely, the purely ideal triangulations for nonempty $\partial M$, and the $1-$ vertex triangulations for closed $M$ ).

Recently, generalized versions of the notion of branching (see the definitions below), with the nice property of existing on every triangulation, have emerged as useful devices to deal with simplicial formulas defined over triangulations equipped with solutions of Thurston's PSL $(2, \mathbb{C}$ ) consistency equations (or variations of them; see Luo and Schlenker [13] and Luo Tillmann and Yang [14]). For instance, motivated by his work in progress on the entropy of solutions of the homogeneous $\operatorname{PSL}(2, \mathbb{R})$ Thurston equations, Luo introduced the notion of $\mathbb{Z} / 2 \mathbb{Z}$-taut structure on a triangulation, and it turns out that a certain notion of weak branching, widely employed below together with the underlying notion of prebranching, easily allows us to show that every triangulation admits $\mathbb{Z} / 2 \mathbb{Z}$-taut structures (see Remark 1.2). As another example, the same notions of weak branching and prebranching were exploited by Baseilhac and the first author in [2] to extend the construction of quantum hyperbolic invariants [4; 3] to an arbitrary hyperbolic one-cusped manifold, over a canonical Zariski-open set of the geometric component of its character variety.

In several instances Luo [12] suggested that a combinatorial encoding of spin structures based on arbitrary triangulations might be of use for the construction of spin-refined invariants obtained from simplicial formulas as those mentioned in the previous paragraph. In this note we provide such a presentation, using the notion of weak branching already alluded to.

The results established in this paper provide an "objects/moves" combinatorial presen- 
tation of the set of all pairs $(M, s)$, with $M$ a compact oriented 3-manifold and $s$ a spin structure on $M$, in the following sense:

- given any (loose) triangulation $\mathcal{T}$ of $M$, with ideal vertices at the components of $\partial M$ and possibly internal vertices, and any $s$, we encode $s$ by decorating $\mathcal{T}$ with certain extra combinatorial structures;

- we exhibit combinatorial moves on decorated triangulations relating to each other any two that encode the same $(M, s)$.

We note that all our moves are explicitly described, but one of them has an intrinsically global nature. On the other hand, in the second part of the paper we will show that this move can actually be replaced, in a suitable sense, by a combination of local ones. This last result is subtle and technically quite demanding, it is based on some nontrivial algebraic constructions, and it unveils unexpected coherence properties of the graphic calculus we use to encode weakly branched triangulations.

A first application of the technology developed in the present note appears in [2], where our results are used to solve a sign indeterminacy in the phase anomaly of the quantum hyperbolic invariants (see Remark 2.9). We also note that adapting the arguments of [6, Chapter 8], the results of this article can be used to provide an effective construction of the Roberts spin-refined Turaev-Viro invariants [19], and of the related Blanchet spin-refined Reshetikhin-Turaev invariants [8] of the double of a manifold.

We conclude this introduction by mentioning that a notion related to that of spin structure, namely that of $\mathrm{Spin}^{\mathrm{c}}$ (or, equivalently, Euler) structure, has also been to some extent combinatorially treated by means of triangulations and special spines; see the authors $[6 ; 7 ; 1]$. However, even in the context of branched spines, a complete objects/moves encoding of $\operatorname{Spin}^{\mathrm{c}}$ structures, in the strict sense of the present paper, has not been fully developed yet. More precisely, sticking for simplicity to the case of a closed manifold $M$, by combining $[6 ; 7 ; 1]$ one has the following:

- every branched triangulation $\mathcal{T}$ of $M$ with only one vertex carries an Euler structure $e_{\mathcal{T}}$ on $M$ and a distinguished Euler $1-$ chain $c_{\mathcal{T}}$ with respect to $\mathcal{T}$, so that $c_{\mathcal{T}}$ encodes $e_{\mathcal{T}}$;

- the "sliding" moves of [6] preserve $e_{\mathcal{T}}$ but in general they do not suffice to relate to each other any two triangulations $\mathcal{T}$ and $\mathcal{T}^{\prime}$ such that $e_{\mathcal{T}}=e_{\mathcal{T}^{\prime}}$;

- for every branched move from $\mathcal{T}$ to $\mathcal{T}^{\prime}$ one can effectively modify $c_{\mathcal{T}}$ to an Euler $1-$ chain $\hat{c}_{\mathcal{T}}$ with respect to $\mathcal{T}^{\prime}$ so that $\hat{c}_{\mathcal{T}}$ encodes $e_{\mathcal{T}}$ as well, and hence establish a posteriori whether $e_{\mathcal{T}}$, coincides with $e_{\mathcal{T}}$ by checking that $c_{\mathcal{T}}-\widehat{c}_{\mathcal{T}}$ is homologically null.

We expect the treatment of Spin ${ }^{c}$ structures by means of arbitrary triangulations and weak branchings to be even more complicated than just described. 


\section{Statements for triangulations}

In this section we state some results that provide in terms of arbitrary triangulations a combinatorial encoding of spin structures on oriented 3-manifolds. The geometric construction underlying this encoding actually employs certain objects called special spines, and will be fully described in Sections 2 and 3. As a matter of fact, triangulations and special spines are equivalent to each other via duality, but perhaps the majority of topologists are more familiar with the language of triangulations, which is why we are anticipating our statements in this section. On the other hand the direct description in terms of triangulations of the vector fields on which our constructions and results are based would be rather awkward, therefore, starting from Section 2, we will always employ special spines.

\subsection{Triangulations, prebranchings and weak branchings}

In this note $M$ will always be a connected, compact and oriented 3-manifold, with or without boundary. We also assume that $\partial M$ has no $S^{2}$ component (otherwise we canonically cap it with $D^{3}$ ). We begin with several definitions. A triangulation of $M$ is the datum $\mathcal{T}$ of

- a finite number of oriented abstract tetrahedra,

- an orientation-reversing simplicial pairing of the 2 -faces of these tetrahedra,

such that the space obtained by first gluing the tetrahedra along the pairings and then removing open stars of the vertices is orientation-preservingly homeomorphic to $M$ with some punctures (open balls removed). Any number of punctures, including zero, is allowed (but a closed $M$ must be punctured at least once).

A branching on an abstract oriented tetrahedron $\Delta$ is an orientation of its edges such that no face of $\Delta$ is a cycle. Equivalently, one vertex of $\Delta$ should be a source and one should be a sink, as illustrated in Figure 1(left). Note that the figure shows the only two possible branched tetrahedra up to oriented isomorphism. They are characterized by an index \pm 1 , to define which one denotes by $v_{j}$ the vertex of $\Delta$ towards which $j$ edges of $\Delta$ point, and one checks whether the ordering $\left(v_{0}, v_{1}, v_{2}, v_{3}\right)$ defines the orientation of $\Delta$ or not. Each face of a branched abstract tetrahedron is endowed with the prevailing orientation induced by its edges. 

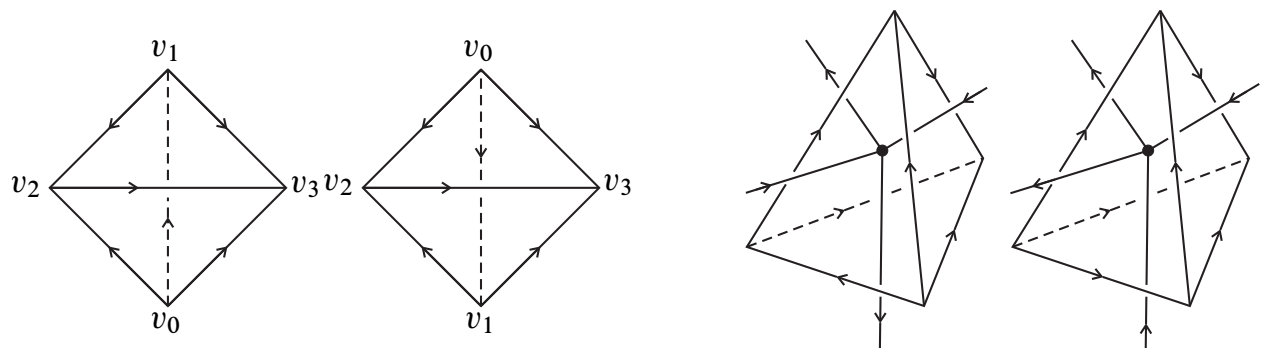

Figure 1: Left: a branched tetrahedron of index +1 and one of index -1 Right: a weak branching compatible with a prebranching
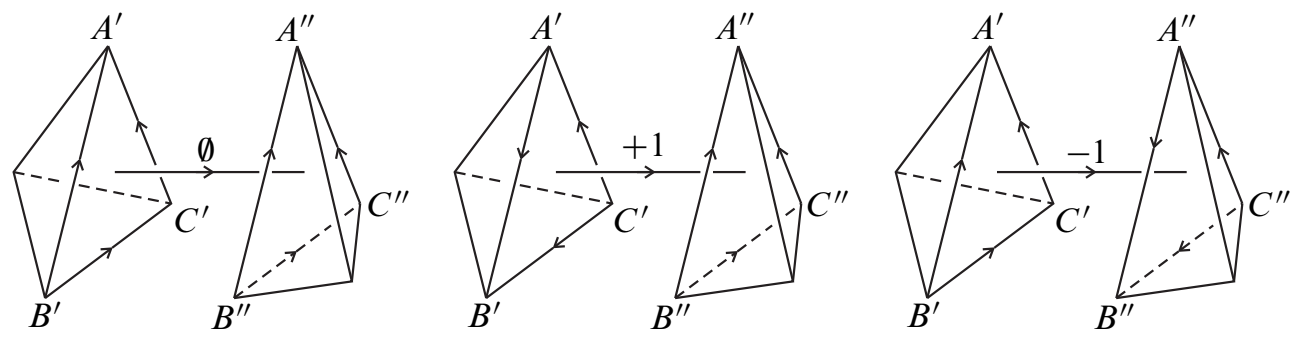

Figure 2: The three types of face pairings in a weakly branched triangulation

A prebranching on a triangulation $\mathcal{T}$ is an orientation $\omega$ of the edges of the gluing graph $\Gamma$ of $\mathcal{T}$ (a 4-valent graph) such at each vertex two edges are incoming and two are outgoing. Given such an $\omega$, a weak branching $b$ compatible with $\omega$ is the choice of an abstract branching for each tetrahedron in $\mathcal{T}$ such that $\Gamma$ with its orientation $\omega$ is positively transversal to each face of each tetrahedron in $\mathcal{T}$, as in Figure 1(right). Note that for such a $b$, when two faces are glued in $\mathcal{T}$, two things can happen:

- all three edge orientations are matched, as in Figure 2(left); to this situation we assign a label $\emptyset$ used below;

- only one edge orientation is matched, as in Figure 2(center/right); moreover, in both the glued faces, the edge whose orientation is matched has the prevailing orientation of the face; taking into account the ambient orientation, this situation arises in two different ways, to which we assign the labels \pm 1 used below.

\subsection{Spin structure from a weak branching and a 1-chain}

All the constructions and results of the rest of this section will be explained and proved in Sections 2 and 3 in the dual context of special spines. Let a triangulation $\mathcal{T}$ with prebranching $\omega$ and compatible weak branching $b$ be given. We will now define a 
chain $\bar{\alpha}(P, \omega, b)=\sum_{e} \alpha(e) \cdot e \in C_{1}(\mathcal{T} ; \mathbb{Z} / 2 \mathbb{Z})$, where $e$ runs over all edges of $\mathcal{T}$. The value of $\alpha(e)$ is the sum of a fixed initial contribution 1 plus certain contributions of two different types; both contribution types are computed in the group $G=\left(\frac{1}{2} \cdot \mathbb{Z}\right) / 2 \mathbb{Z}$, but for each of them the sum is in $\mathbb{Z} / 2 \mathbb{Z}$; here is the description of the two types:

- endow $e$ with an arbitrary orientation and in the abstract tetrahedra of $\mathcal{T}$, consider the collection of all the edges projecting to $e$ and of type $v_{0} v_{2}$ or $v_{1} v_{3}$; for each such abstract edge $\tilde{e}$ take a contribution $+\frac{1}{2}$ or $-\frac{1}{2}$ depending on whether the projection from $\tilde{e}$ to $e$ preserves or reverses the orientation;

- consider all the face gluings as in Figure 2 in which $e$ is involved (with multiplicity) and take a contribution depending as follows on the type $t$ of the gluing and on the position of $e$ within it:

$\triangleright \quad 0$ if $t=\emptyset$, regardless of the position of $e$;

$\triangleright \quad 1$ if $t= \pm 1$ and the orientation of $e$ is matched by the gluing;

$\triangleright \mp \frac{1}{2}$ if $t= \pm 1$ and the orientation of $e$ is not matched by the gluing.

Proposition 1.1 The chain $\bar{\alpha}(P, \omega, b)$ is a boundary, and to every $\bar{\beta} \in C_{2}(\mathcal{T} ; \mathbb{Z} / 2 \mathbb{Z})$ such that $\partial \bar{\beta}=\bar{\alpha}(P, \omega, b)$ there corresponds a spin structure $s(\mathcal{T}, \omega, b, \bar{\beta})$ on $M$. Moreover $s\left(\mathcal{T}, \omega, b, \bar{\beta}_{0}\right)=s\left(\mathcal{T}, \omega, b, \bar{\beta}_{1}\right)$ if and only if $\bar{\beta}_{0}+\bar{\beta}_{1}$ is 0 in $H_{2}(\mathcal{T} ; \mathbb{Z} / 2 \mathbb{Z})$.

Remark 1.2 Let $b$ be a weak branching compatible with a prebranching $\omega$ on a triangulation $\mathcal{T}$ of a manifold $M$. If in each abstract tetrahedron of $\mathcal{T}$ we choose the pair of opposite edges of types $v_{0} v_{2}$ and $v_{1} v_{3}$ with respect to $b$, then the choice actually depends on $\omega$ only, not on $b$. Moreover one sees that for all edges $e$ of $\mathcal{T}$ in $M$ there is always an even number of abstract edges of types $v_{0} v_{2}$ or $v_{1} v_{3}$ projecting to $e$ (this corresponds to the fact that the contributions to $\bar{\alpha}(P, \omega, b)$ of the first type described above are in $\mathbb{Z} / 2 \mathbb{Z}$, and it is established in Proposition 2.7 below). It follows that, giving sign -1 to all the abstract edges $v_{0} v_{2}$ and $v_{1} v_{3}$, and sign +1 to the other edges, we get a $\mathbb{Z} / 2 \mathbb{Z}$-taut structure on $\mathcal{T}$, as mentioned in the introduction.

\subsection{Triangulation moves preserving the spin structure}

The next results provide the combinatorial encoding of spin structures announced in the title of the paper. From now on all chains $\bar{\beta} \in C_{2}(\mathcal{T} ; \mathbb{Z} / 2 \mathbb{Z})$ will be viewed up to 2-boundaries, without explicit mention.

Proposition 1.3 We have $s\left(\mathcal{T}, \omega, b_{0}, \bar{\beta}_{0}\right)=s\left(\mathcal{T}, \omega, b_{1}, \bar{\beta}_{1}\right)$ if and only if we have that $\left(b_{0}, \bar{\beta}_{0}\right)$ and $\left(b_{1}, \bar{\beta}_{1}\right)$ are related by the moves of Figure 3 (and their compositions and inverses). 

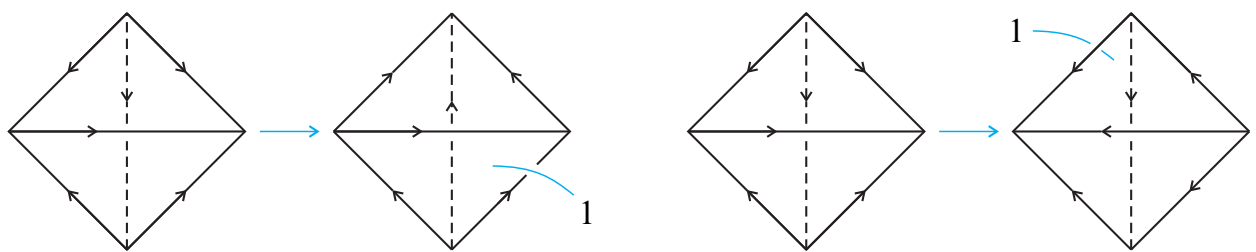

Figure 3: Moves preserving the prebranching and the associated spin structure. In both moves the " 1 " means that 1 must be added to the coefficient in $\bar{\beta}$ of the triangle to which " 1 " is attached; note that in both moves it is the only one whose three edges all retain their orientation under the move.

Proposition 1.4 We have $s\left(\mathcal{T}, \omega_{0}, b_{0}, \bar{\beta}_{0}\right)=s\left(\mathcal{T}, \omega_{1}, b_{1}, \bar{\beta}_{1}\right)$ if and only if we have that $\left(\omega_{0}, b_{0}, \bar{\beta}_{0}\right)$ and $\left(\omega_{1}, b_{1}, \bar{\beta}_{1}\right)$ are related by the moves of Proposition 1.3 and additional moves $(\mathcal{T}, \omega, b, \bar{\beta}) \mapsto\left(\mathcal{T}, \omega^{\prime}, b^{\prime}, \bar{\beta}^{\prime}\right)$ described as follows:

- in the gluing graph of $\mathcal{T}$ (which is oriented by $\omega$ ) take an oriented simple circuit $\gamma$ such that, for each tetrahedron it visits, the two faces it visits share the edge $v_{2} v_{3}$ with respect to $b$, as in Figure 4;

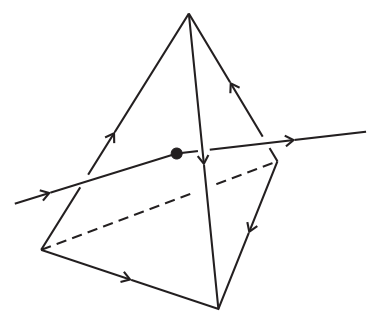

Figure 4: A circuit $\gamma$ in the gluing graph that in each tetrahedron visits faces sharing the edge $v_{2} v_{3}$. The gluing encoded by an edge of $\gamma$ need not match edges of type $v_{2} v_{3}$ to each other.

- define $\omega^{\prime}$ by reversing $\gamma$, define $b^{\prime}$ by reversing each edge $v_{2} v_{3}$ in each tetrahedron visited by $\gamma$, and define $\bar{\beta}^{\prime}$ by adding 1 to the coefficient of each face of $\mathcal{T}$ visited by $\gamma$ and incident to tetrahedra of distinct indices.

Proposition 1.5 We have $s\left(\mathcal{T}_{0}, \omega_{0}, b_{0}, \bar{\beta}_{0}\right)=s\left(\mathcal{T}_{1}, \omega_{1}, b_{1}, \bar{\beta}_{1}\right)$ if and only if the quadruples $\left(\mathcal{T}_{0}, \omega_{0}, b_{0}, \bar{\beta}_{0}\right)$ and $\left(\mathcal{T}_{1}, \omega_{1}, b_{1}, \bar{\beta}_{1}\right)$ are related by the moves of Propositions 1.3 and 1.4 and those shown in Figures 5 and 6.

Remark 1.6 In this result one can avoid the move of Figure 6 if $\mathcal{T}_{0}$ and $\mathcal{T}_{1}$ have the same number of internal vertices and both consist of at least two tetrahedra. 


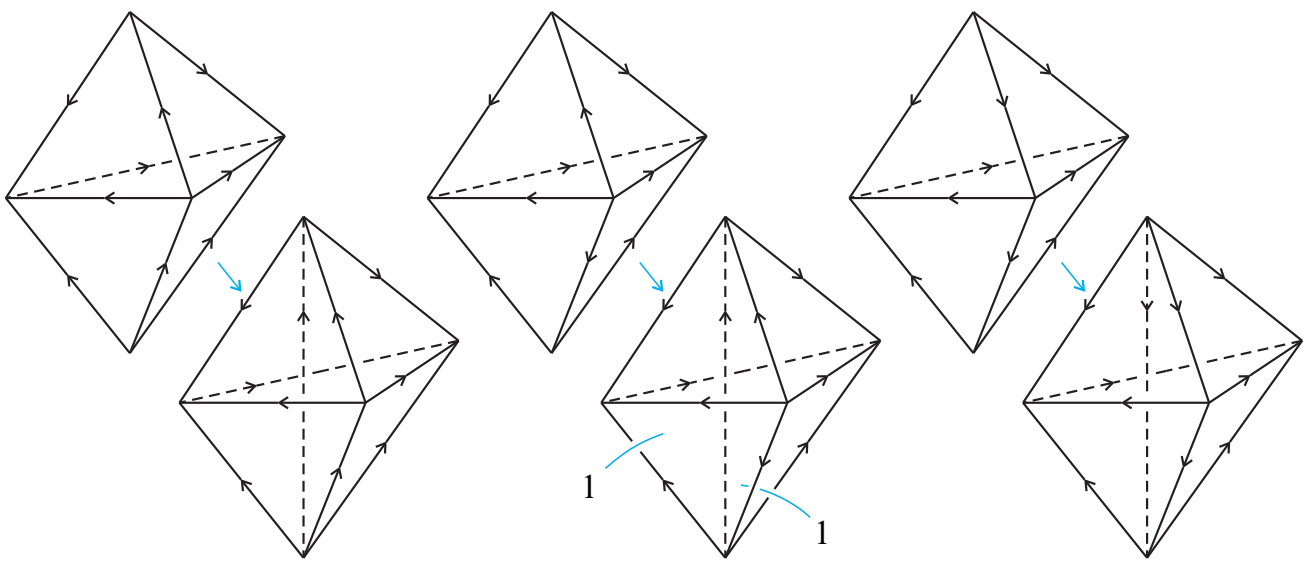

Figure 5: Moves preserving the spin structure. Note that in the central move the coefficients 1 are given to one internal and to one external face; coefficients 0 are never shown.
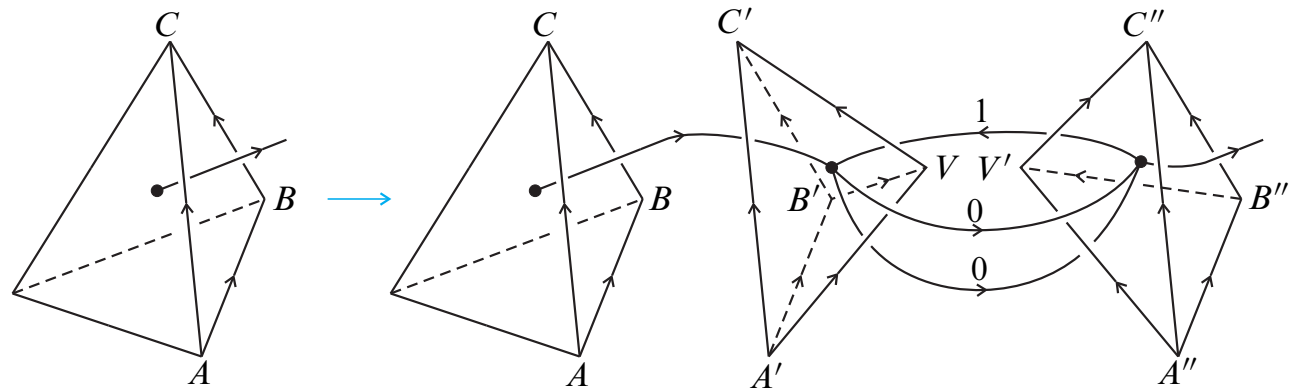

Figure 6: A move increasing by one the number of punctures and preserving the spin structure. The coefficients of $A B V, A C V$ and $B C V$ in the 2-chain after the move are 0,0 and 1 .

\section{Spin structures from weakly branched spines}

We will now explain how the spin structure $s(\mathcal{T}, \omega, b, \bar{\beta})$ mentioned in the previous section is constructed. As announced, this employs the viewpoint of special spines, which is dual to that of triangulations.

To a triangulation $\mathcal{T}$ of $M$ we can associate the dual special spine $P$ of $M$ minus some balls, as suggested in Figure 7. The polyhedron $P$ is a compact 2-dimensional one onto which $M$ minus some balls collapses. Every point of $P$ has a neighbourhood homeomorphic to the cone over a circle, or over a circle with a diameter (in which case the point is said to belong to a singular edge), or over a circle with three radii (in 
which case the point is called a singular vertex, and the neighbourhood itself is called a butterfly). Moreover $P$ has vertices, its singular set $S(P)$ is a 4-valent graph (actually, it is the gluing graph of $\mathcal{T}$ ) and the components of $P$ minus $S(P)$, that we call regions, are homeomorphic to open discs. Any such $P$ is called a special polyhedron, and it is known that there can exist at most one thickening of $P$, namely a punctured manifold $M$ collapsing onto $P$, in which case $P$ dually defines a triangulation of $M$. Moreover one can add to $P$ an easy extra combinatorial structure, called a screw-orientation (see the authors [5]) ensuring that $P$ is thickenable and that its thickening is oriented. A screw-orientation for $P$ is an orientation of each edge $e$ of $P$ and a cyclic ordering of the three germs of regions incident to $e$, up to simultaneous reversal of both, with obvious compatibility at vertices. All the special polyhedra we will consider will be embedded in an oriented 3-manifold or locally embedded in 3-space, and we stipulate from now on that the screw-orientation will always be the induced one, which allows us to avoid discussing screw-orientation and orientation altogether.

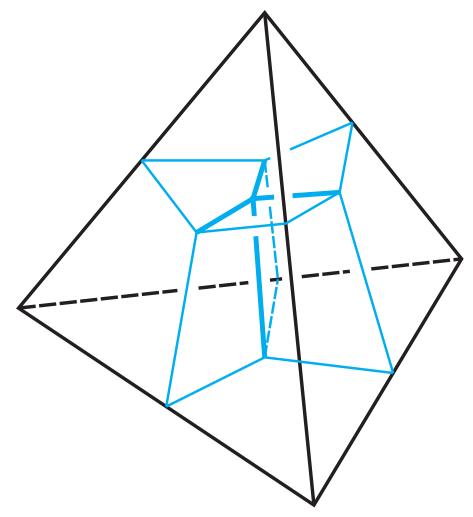

Figure 7: Duality between a tetrahedron and a butterfly (the regular neighbourhood of a vertex in a special spine)

\subsection{Branched spines}

If an oriented tetrahedron $\Delta$ is branched, one can endow each wing of the dual butterfly $Y$ with the orientation such that the edge of $\Delta$ dual to the wing is positively transversal to the wing. (Note that the ambient orientation is used here.) One can moreover smoothen $Y$ along its singular set so that the positive transversal directions to the wings match, as shown in Figure 8, where we show the butterflies dual to the branched tetrahedra of Figure 1(left). We can further define along the singular set of $Y$ two vector fields $v$ (the positive transversal to the wings) and $\mu_{0}$ (the descending vector field), and an orientation of the 4 singular edges of the butterfly, as shown in 
Figure 9. Note that the orientation of an edge $e$ of a butterfly is always given by the wedge of $v$ and $\mu_{0}$ along $e$, and it is the prevailing orientation of the three induced by the wings incident to $e$.
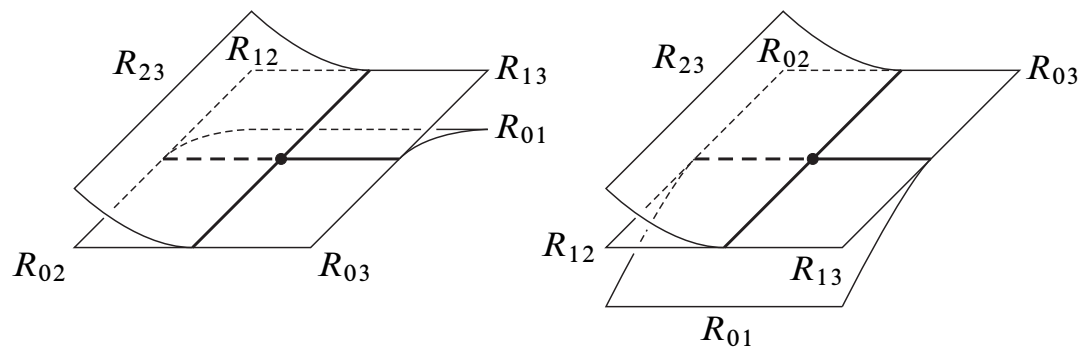

Figure 8: Smoothing of a butterfly carried by a branching of its dual tetrahedron $\Delta$. Here $R_{i j}$ denotes the wing of the butterfly dual to the edge $v_{i} v_{j}$ of $\Delta$.
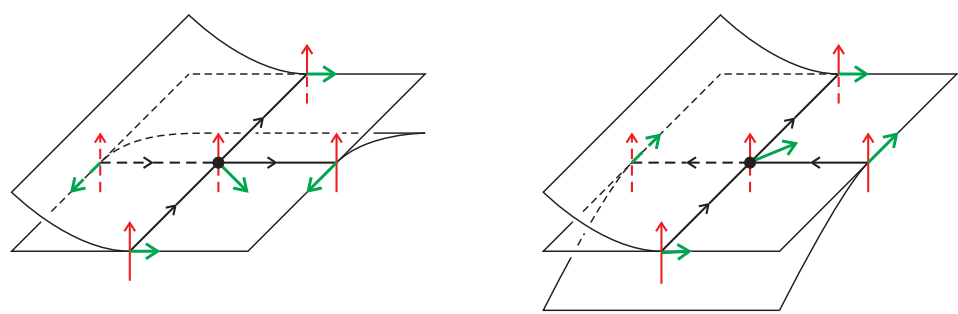

Figure 9: The fields $v$ (vertical) and $\mu_{0}$ (horizontal) along the singular set of a smooth butterfly, and the orientation of its edges

\subsection{Weakly branched triangulations and the induced frame along the dual 1-skeleton}

Let us fix in this subsection a triangulation $\mathcal{T}$ of an oriented manifold $M$ and the special spine $P$ dual to $\mathcal{T}$. If $\mathcal{T}$ carries a global branching, namely if each tetrahedron in $\mathcal{T}$ is endowed with a branching so that all face pairings match the edge orientations, then the frame $\left(v, \mu_{0}\right)$ extends to $S(P)$, as in Figure 10 below. However, a global branching does not always exist, and we explain here how the structure of weak branching still allows to globally define a frame along $S(P)$.

Remark 2.1 We will call frame on a subset $X$ of $M$ a pair of linearly independent sections defined on $X$ of the tangent bundle $T M$ of $M$; since $M$ is oriented, this uniquely induces a trivialization of $T M$ on $X$. 
Let us then take a prebranching $\omega$ of $\mathcal{T}$, viewed as an orientation of $S(P)$ with two incoming and two outgoing edges at each vertex, and a weak branching $b$ compatible with $\omega$. For an edge $e$ of $P$ the following three possibilities (corresponding to those in Figure 2) occur.

- The edge $e$ can be a branched edge (type $\emptyset$ ), namely one along which the branchings defined at the ends are compatible, as in Figure 10; the same figure shows how to (obviously) extend the frame $\left(\nu, \mu_{0}\right)$ along such an $e$.

- If $e$ is not branched there is only one region $A$ incident to $e$ lying on the two-fold side (namely, to the left of $e$ ) at both ends of $e$, and we say that

$\triangleright \quad e$ is a positive unbranched edge (type +1 ) if $A$ is under at the beginning of $e$ and over at the end of $e$, as in Figure 11(top/left),

$\triangleright \quad e$ is a negative unbranched edge (type -1 ) if $A$ is over at the beginning of $e$ and under at the end of $e$, as in Figure 11(top/right).

In both cases we can again coherently define $v$ along $e$, by letting the transverse orientation of $A$ prevail on the other two, and accordingly define $\mu_{0}$, as illustrated in Figure 11(bottom).

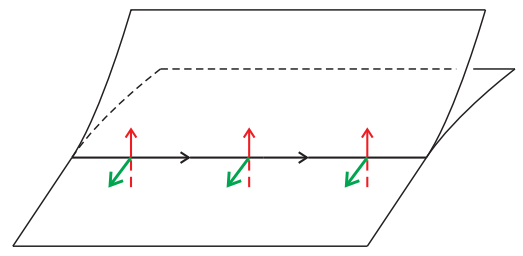

Figure 10: A branched edge and the extension of $\left(\nu, \mu_{0}\right)$ along it

For a technical but important reason, to a spine $P$ with prebranching $\omega$ and compatible weak branching $b$ we actually associate a frame $\varphi=(\nu, \mu)$ that is obtained from the above described $\left(v, \mu_{0}\right)$ by adding to $\mu_{0}$ a full rotation around $v$ along each unbranched edge of $P$, as shown in Figure 12. We summarize the main points of our construction in the following.

Definition 2.2 Let $\mathcal{T}$ be a triangulation of a compact oriented 3-manifold $M$, and let $P$ be the dual spine of $M$ minus some balls. A prebranching on $P$ is an orientation $\omega$ of its edges such that at each vertex two germs of edges are incoming and two are outgoing. A weak branching on $\mathcal{T}$ compatible with $\omega$ is a choice $b$ of a branching for each tetrahedron of $\mathcal{T}$, such that $b$ induces $\omega$ at each vertex of $P$ according to Figure 9. The frame $\varphi(P, \omega, b)=(\nu, \mu)$ defined along $S(P)$ is given by the pair $\left(\nu, \mu_{0}\right)$ at the vertices of $P$ as in Figure 9 , with extension $\left(v, \mu_{0}\right)$ along the edges 

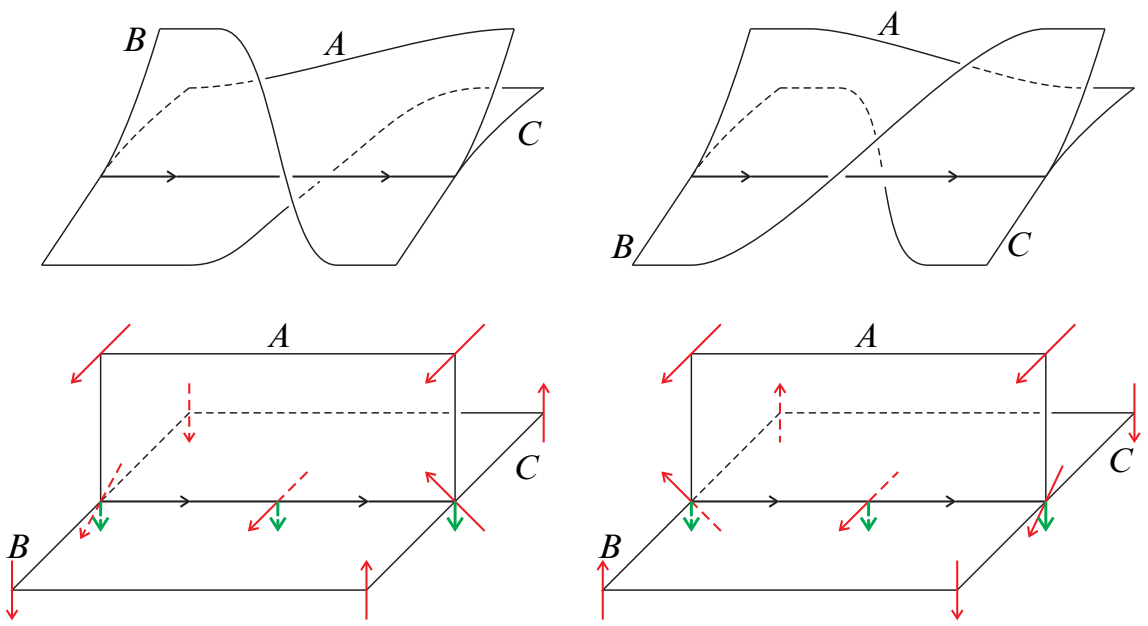

Figure 11: Top left: a positive unbranched edge Top right: a negative one Bottom: the corresponding extensions of $\left(\nu, \mu_{0}\right)$
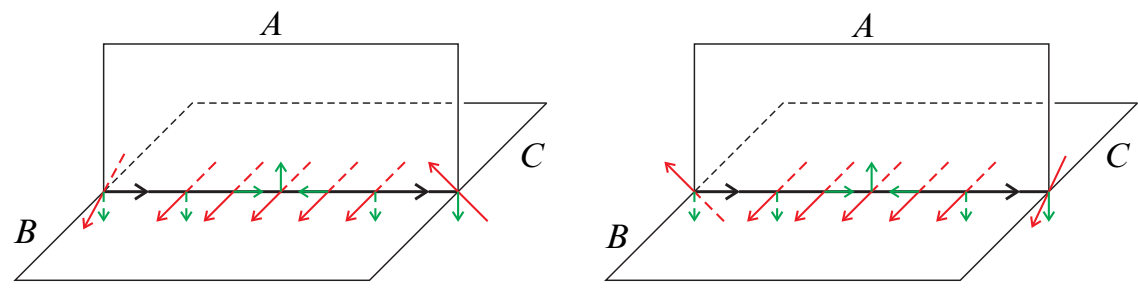

Figure 12: The field $\mu$ obtained by adding a full rotation to $\mu_{0}$ along each (positive or negative) unbranched edge of $P$

of $P$ as in Figures 10 (branched edges) and 11 (unbranched edges), and correction from $\left(v, \mu_{0}\right)$ to $(v, \mu)$ along the unbranched edges as in Figure 12.

Remark 2.3 For every triangulation $\mathcal{T}$ the dual spine $P$ always admits some prebranching $\omega$. Given $\omega$, for a compatible weak branching $b$ there are 4 independent choices at each tetrahedron of $\mathcal{T}$. The frame $\varphi(P, \omega, b)$ is well-defined up to homotopy on $S(P)$.

\subsection{Graphs representing weakly branched triangulations}

In this subsection we introduce a convenient graphic encoding for weakly branched triangulations that we will later use to prove (the dual version of) Proposition 1.1. Let $\mathcal{N}$ be the set of finite 4 -valent graphs $\Gamma$ with the following extra structures: 

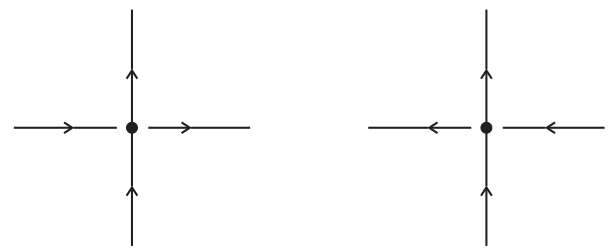

Figure 13: Planar structure of index +1 (left) or -1 (right) at a vertex of a graph in $\mathcal{N}$

- each edge of $\Gamma$ is oriented and bears a color $\emptyset,+1$ or -1 ;

- at each vertex of $\Gamma$ a planar structure as in Figure 13 left/right is given.

Let $\mathcal{T}$ be a weakly branched triangulation of an oriented 3-manifold $M$, and let $P$ be the dual spine of $M$ minus some balls. We can turn $S(P)$ into a graph $\Gamma(\mathcal{T}) \in \mathcal{N}$ by associating to a branched tetrahedron of $\mathcal{T}$ as in Figure 1(left) (or to a smoothed vertex of the dual spine $P$ as in Figure 9) a vertex as in Figure 13, and giving color $\emptyset,+1,-1$ to each edge depending on its type.

The procedure just described can of course be reversed, namely to a graph $\Gamma \in \mathcal{N}$ we can associate a weakly branched triangulation $\mathcal{T}(\Gamma)$ of an oriented manifold $M$. Some examples of how to explicitly construct the spine $P$ dual to $\mathcal{T}(\Gamma)$ along the edges of $\Gamma$ are illustrated in Figure 14. (Recall that $P$ is determined by the attaching
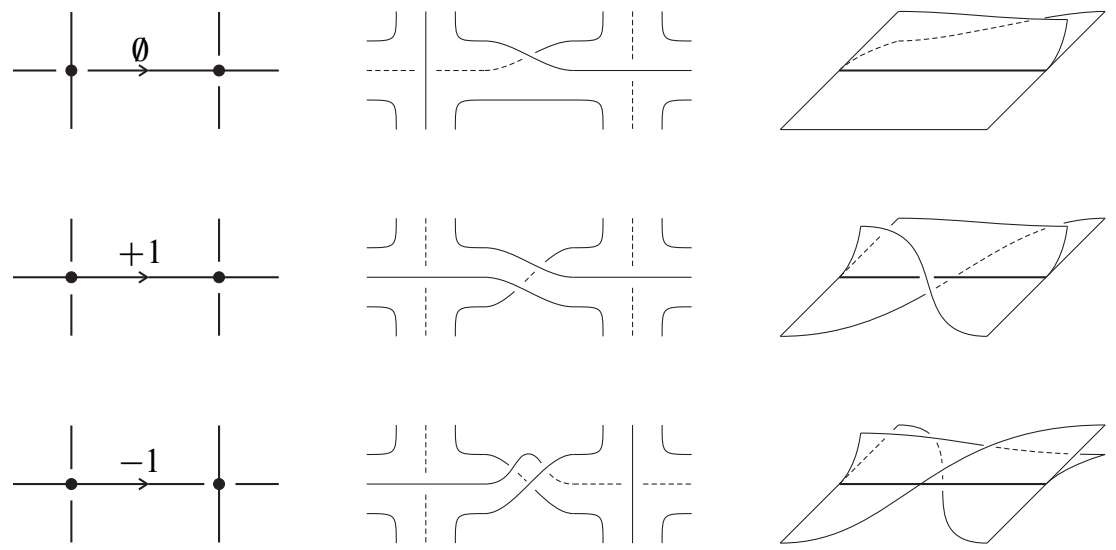

Figure 14: Reconstruction of a weakly branched spine from a graph in $\mathcal{N}$

circles of its regions to $S(P)$, which is what we show in Figure 14(centre), and that the screw-orientation of $P$ is induced by the local embedding in 3-space, shown in Figure 14(right).) We summarize our construction as follows. 
Proposition 2.4 The set $\mathcal{N}$ of decorated graphs corresponds bijectively to the set of triples $(P, \omega, b)$ with $P$ an oriented special spine, $\omega$ a prebranching on $P$ and $b$ a weak branching compatible with $\omega$.

For later purpose we now need to extend the set of graphs $\mathcal{N}$ to some $\widetilde{\mathcal{N}}$, by allowing 2 -valent vertices besides the 4-valent (decorated) ones, and insisting that the edge orientations should match through the 2 -valent vertices. By interpreting each 2 -valent vertex as $\equiv$ or $\overline{\overline{-}}$ we can then associate as above to each element $\widetilde{\Gamma}$ of $\widetilde{\mathcal{N}}$ a weakly branched special spine. On the other hand we can define the fusion of two edges separated by a valence- 2 vertex by interpreting the set of colors $\{\emptyset,+1,-1\}$ as $\mathbb{Z} / 3 \mathbb{Z}$ and postulating that colors sum up under fusion. Applying fusion as long as possible to $\widetilde{\Gamma} \in \widetilde{\mathcal{N}}$ we then get some $\Gamma \in \mathcal{N}$. The following result can be easily verified; see Figure 15 .
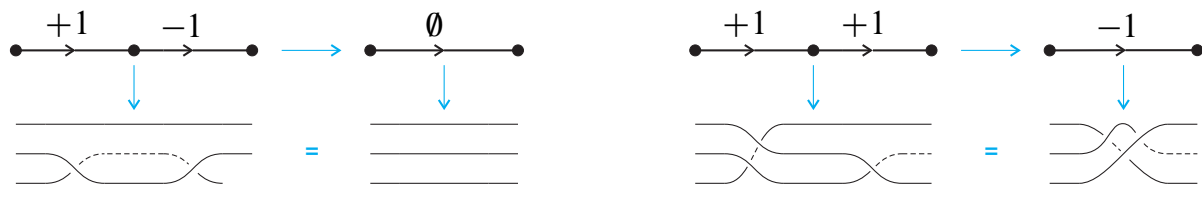

Figure 15: To each $\widetilde{\Gamma} \in \widetilde{\mathcal{N}}$ one can uniquely associate a weakly branched special spine, also given by the graph $\Gamma \in \mathcal{N}$ obtained from $\widetilde{\Gamma}$ by fusing the edges through valence- 2 vertices.

Proposition 2.5 The weakly branched special spine associated to $\widetilde{\Gamma} \in \widetilde{\mathcal{N}}$ is independent of the interpretation of the $2-v a l e n t$ vertices, and it coincides with the spine corresponding to the graph $\Gamma \in \mathcal{N}$ obtained from $\widetilde{\Gamma}$ by edge-fusion.

We conclude this subsection by explaining why have defined $\varphi(P, \omega, b)=(\nu, \mu)$ not simply as $\left(\nu, \mu_{0}\right)$, but adding instead a full twist to $\mu_{0}$ along unbranched edges.

Proposition 2.6 Take $\widetilde{\Gamma} \in \widetilde{\mathcal{N}}$ and let $\Gamma \in \mathcal{N}$ be obtained from $\widetilde{\Gamma}$ by fusing edges through valence- 2 vertices. Then the frames $(\widetilde{v}, \tilde{\mu})$ and $(v, \mu)$ carried by $\widetilde{\Gamma}$ and by $\Gamma$ are homotopic to each other.

Proof We have to show that when we fuse two colored edges into one the frame $(\nu, \mu)$ defined by the fusion is homotopic to the concatenation of the frames defined by the two edges. Recall that the color of the combination is the sum of the colors, and note that the conclusion is obvious when one of the edge colors is $\emptyset$. When the two edge colors are opposite to each other one can examine Figure 11 and see that the 
concatenation of the two frames $\left(\nu, \mu_{0}\right)$ is homotopic to a constant frame; at the level of $(\nu, \mu)$ we would have to add two full twists to $\mu_{0}$, which amounts to nothing, and the conclusion follows. We are left to deal with the sum of two edges with identical color. We deal with the case $+1+1$, since $-1-1$ is similar. The frames $\left(v, \mu_{0}\right)$ corresponding to $+1+1$ and to -1 are shown in Figure 16, and recognized to differ by a full twist. When passing to $(\nu, \mu)$ we have to add two full twists to $\mu$ (that is, nothing) in the $+1+1$ configuration, and one full twist in the -1 configuration, thus getting homotopic frames.

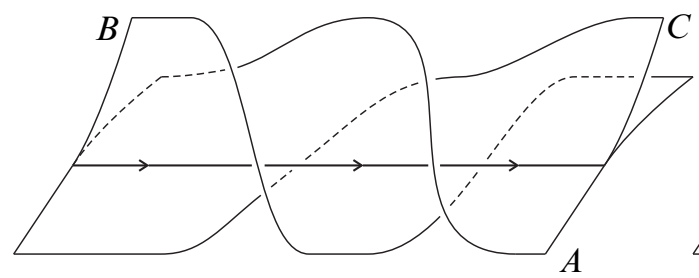

A

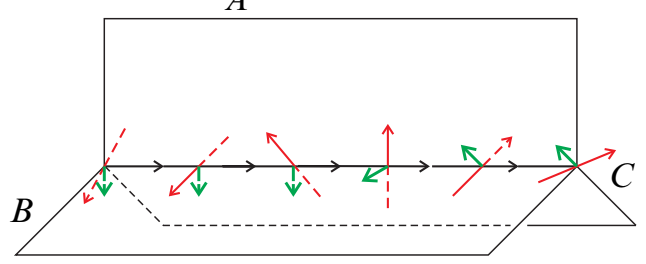

Figure 16: The frames $\left(v, \mu_{0}\right)$ corresponding to $+1+1$ and to -1

\subsection{Obstruction computation}

We now denote by $\alpha(P, \omega, b) \in C^{2}(P ; \mathbb{Z} / 2 \mathbb{Z})$ the obstruction to extending $\varphi(P, \omega, b)$ to a frame defined on $P$. To define $\alpha(P, \omega, b)$, note that $T M$ can always be trivialized as $\mathrm{GL}^{+}(3 ; \mathbb{R}) \times R$ on each open region $R$ of $P$, and $\alpha(P, \omega, b)(R)$ is the element of $\pi_{1}\left(\mathrm{GL}^{+}(3 ; \mathbb{R})\right)=\mathbb{Z} / 2 \mathbb{Z}$ represented by the restriction of $\varphi(P, \omega, b)$ to (a loop parallel to) $\partial R$. The next result shows that the chain $\bar{\alpha}(P, \omega, b)=\sum_{e} \alpha(e) \cdot e \in C_{1}(\mathcal{T} ; \mathbb{Z} / 2 \mathbb{Z})$ introduced in Section 1 is dual to $\alpha(P, \omega, b)$, namely that $\alpha(e)=\alpha(P, \omega, b)(R)$ if $R$ is the region of $P$ dual to an edge $e$ of $\mathcal{T}$.

Proposition 2.7 Given $\Gamma \in \mathcal{N}$ decorate the attaching circles of the regions of the special spine $P$ defined by $\Gamma$ as follows:

- at each vertex of $\Gamma$ put arrows as in Figure 17(top); 


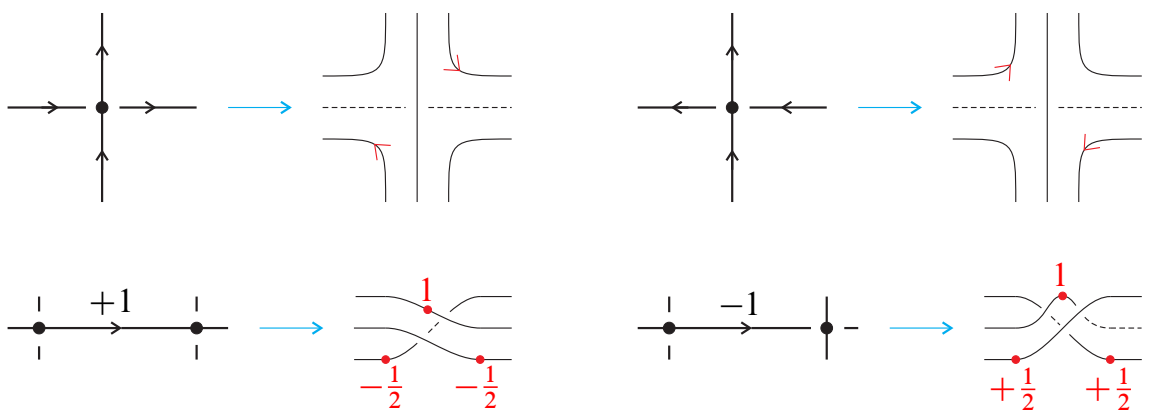

Figure 17: Decoration of the attaching circles of the regions near vertices and edges

- at each edge $e$ of $\Gamma$, if the edge color is $\emptyset$, put nothing, while if the edge color is \pm 1 put a weight 1 on the region that lies to the left of $e$ at both ends of $e$, and $\mp \frac{1}{2}$ on the two other regions (see two examples in Figure 17(bottom)).

Then $\alpha(P, \omega, b)(R) \in \mathbb{Z} / 2 \mathbb{Z}$ is computed as 1 plus the sum of the numerical contributions along $\partial R$ plus the sum of contributions from arrows, turned numerical as follows: choose for $\partial R$ an arbitrary orientation and give each arrow value $+\frac{1}{2}$ or $-\frac{1}{2}$ depending on whether it agrees or not with the orientation. Moreover, both the sum of the numerical contributions and that of the contributions from arrows turned numerical belong to $\mathbb{Z} / 2 \mathbb{Z}$.

Proof Recall first that $\varphi(P, \omega, b)=(\nu, \mu)$ is obtained from $\left(\nu, \mu_{0}\right)$ by adding a full twist to $\mu_{0}$ along the edges of $P$ having color \pm 1 . It is then sufficient to show that the obstruction $\alpha_{0}$ to extending $\left(\nu, \mu_{0}\right)$ is computed by decorating the regions of $P$ as in Figure 17(top) near the vertices and as in Figure 18 near the edges.
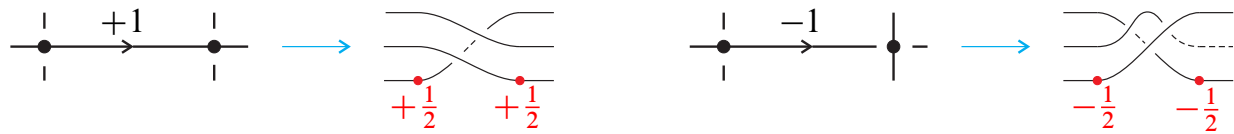

Figure 18: Reduced decoration near edges, used to compute $\alpha_{0}$

Let us now pick a region $R$, give it some orientation, and compute $\alpha_{0}(R)$. Thanks to the orientation of $R$ and of the ambient manifold $M$, for a vector at some point of $\partial R$ the positions shown in Figure 19 are well-defined. We now analyze how the positions of $v$ and $\mu_{0}$ change as $\partial R$ travels near a vertex or edge of $P$.

From Figure 9 one sees that $\left(\nu, \mu_{0}\right)$ does not change at a vertex except if $\partial R$ is in one of the two positions indicated by arrows in Figure 17(top) (the sink and the source quadrants of the vertex); for these, we have 4 different possibilities, two as follows: 


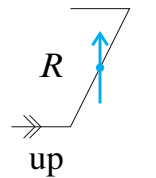

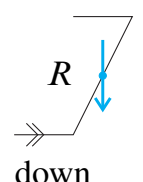

down

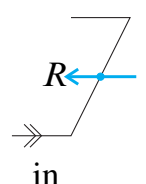

in

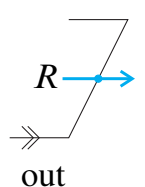

out

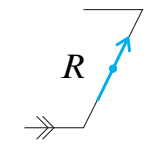

for(ward)

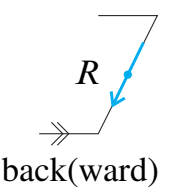

Figure 19: Positions of a vector on the boundary of an oriented region

\begin{tabular}{c|c||c|c} 
& position of $R$ & & position of $R$ \\
\hline$V_{1}$ & sink quadrant of vertex & $V_{2}$ & source quadrant of the vertex
\end{tabular}

with $\partial R$ oriented as the arrow in Figure 17(top), and two more $\bar{V}_{1}$ and $\bar{V}_{2}$ with opposite orientation of $\partial R$; the corresponding changes of $\nu$ and $\mu_{0}$ are

\begin{tabular}{c|c|c||c|c|c} 
& $\Delta v$ & $\Delta \mu_{0}$ & & $\Delta v$ & $\Delta \mu_{0}$ \\
\hline \hline$V_{1}$ & up $\rightarrow$ up $\rightarrow$ up & out $\rightarrow$ back $\rightarrow$ in & $\bar{V}_{1}$ & down $\rightarrow$ down $\rightarrow$ down & in $\rightarrow$ for $\rightarrow$ out \\
\hline$V_{2}$ & up $\rightarrow$ up $\rightarrow$ up & in $\rightarrow$ for $\rightarrow$ out & $\bar{V}_{2}$ & down $\rightarrow$ down $\rightarrow$ down & out $\rightarrow$ back $\rightarrow$ in
\end{tabular}

and this description applies whatever the index of the vertex.

Turning to $\Delta\left(v, \mu_{0}\right)$ along an edge $e$, of course nothing happens if $e$ is branched or $e$ is unbranched but $R$ is in position $A$ in Figure 11; otherwise we have 8 possibilities, 4 with $\partial R$ concordant with $e$ and $R$ in the following position:

\begin{tabular}{c|c||c|c} 
& position of $R$ & & position of $R$ \\
\hline \hline$E_{1}$ & $B$ in Figure 11(left) & $E_{2}$ & $C$ in Figure 11(left) \\
\hline$E_{3}$ & $B$ in Figure 11(right) & $E_{4}$ & $C$ in Figure 11(right)
\end{tabular}

and 4 more $\bar{E}_{j}$ with $\partial R$ discordant with $e$; the corresponding $\Delta\left(v, \mu_{0}\right)$ is:

\begin{tabular}{c|c|c||c|c|c} 
& $\Delta v$ & $\Delta \mu_{0}$ & & $\Delta v$ & $\Delta \mu_{0}$ \\
\hline \hline$E_{1}$ & up $\rightarrow$ in $\rightarrow$ down & out $\rightarrow$ up $\rightarrow$ in & $\bar{E}_{1}$ & up $\rightarrow$ in $\rightarrow$ down & in $\rightarrow$ down $\rightarrow$ out \\
\hline$E_{2}$ & down $\rightarrow$ out $\rightarrow$ up & in $\rightarrow$ down $\rightarrow$ out & $\bar{E}_{2}$ & down $\rightarrow$ out $\rightarrow$ up & out $\rightarrow$ up $\rightarrow$ in \\
\hline$E_{3}$ & down $\rightarrow$ in $\rightarrow$ up & in $\rightarrow$ up $\rightarrow$ out & $\bar{E}_{3}$ & down $\rightarrow$ in $\rightarrow$ up & out $\rightarrow$ down $\rightarrow$ in \\
\hline$E_{4}$ & up $\rightarrow$ out $\rightarrow$ down & out $\rightarrow$ down $\rightarrow$ in & $\bar{E}_{4}$ & up $\rightarrow$ out $\rightarrow$ down & in $\rightarrow$ up $\rightarrow$ out
\end{tabular}

The value of $\alpha_{0}(R)$ will be given in $\pi_{1}\left(\mathrm{GL}^{+}(3 ; \mathbb{R})\right)=\mathbb{Z} / 2 \mathbb{Z}=\{0,1\}$ by 1 plus some contribution of each configuration $V_{i}, \bar{V}_{i}, E_{j}, \bar{E}_{j}$, but

- the $V_{i}, \bar{V}_{i}, E_{j}, \bar{E}_{j}$ cannot appear in arbitrary order: only some concatenations are possible; 
- the individual $V_{i}, \bar{V}_{i}, E_{j}, \bar{E}_{j}$ do not make sense in $\pi_{1}\left(\mathrm{GL}^{+}(3 ; \mathbb{R})\right)$ but some of their concatenations do, when $\left(v, \mu_{0}\right)$ is the same at the two ends of the configuration.

The idea of the proof is then to assign to each $V_{i}, \bar{V}_{i}, E_{j}, \bar{E}_{j}$ a value $\pm \frac{1}{2}$ so that, whatever concatenation is possible and makes sense in $\pi_{1}\left(\mathrm{GL}^{+}(3 ; \mathbb{R})\right)$, its geometrically correct value in $\pi_{1}\left(\mathrm{GL}^{+}(3 ; \mathbb{R})\right)$ is the sum of the values of the $V_{i}, \bar{V}_{i}, E_{j}, \bar{E}_{j}$ appearing in it. Turning to the details, the possible concatenations are

$$
\begin{array}{ll}
\left\{V_{1}, \bar{E}_{2}, \bar{E}_{3}\right\}+\left\{V_{2}, \bar{E}_{1}, \bar{E}_{4}\right\}, & \left\{V_{2}, E_{2}, E_{3}\right\}+\left\{V_{1}, E_{1}, E_{4}\right\}, \\
\left\{\bar{V}_{1}, \bar{E}_{1}, \bar{E}_{4}\right\}+\left\{\bar{V}_{2}, \bar{E}_{2}, \bar{E}_{3}\right\}, & \left\{\bar{V}_{2}, E_{1}, E_{4}\right\}+\left\{\bar{V}_{1}, E_{2}, E_{3}\right\},
\end{array}
$$

and some concatenations that readily make sense in $\pi_{1}\left(\mathrm{GL}^{+}(3 ; \mathbb{R})\right)$ are

$$
\begin{array}{ll}
V_{1}+V_{2}=V_{2}+V_{1}=1, & \bar{V}_{1}+\bar{V}_{2}=\bar{V}_{2}+\bar{V}_{1}=1, \\
E_{1}+E_{2}=E_{2}+E_{1}=1, & \bar{E}_{1}+\bar{E}_{2}=\bar{E}_{2}+\bar{E}_{1}=1, \\
E_{1}+E_{3}=E_{3}+E_{1}=0, & \bar{E}_{1}+\bar{E}_{3}=\bar{E}_{3}+\bar{E}_{1}=0, \\
E_{2}+E_{4}=E_{4}+E_{2}=0, & \bar{E}_{2}+\bar{E}_{4}=\bar{E}_{4}+\bar{E}_{2}=0, \\
E_{3}+E_{4}=E_{4}+E_{3}=1, & \bar{E}_{3}+\bar{E}_{4}=\bar{E}_{4}+\bar{E}_{3}=1 ;
\end{array}
$$

see for instance Figure 20 for $E_{1}+E_{2}=1$, where the concatenation is shown on the left and then homotoped to $1 \in \pi_{1}\left(\mathrm{GL}^{+}(3 ; \mathbb{R})\right)$.

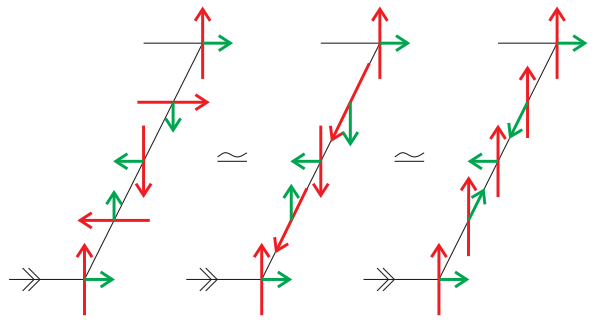

Figure 20: Proof that $E_{1}+E_{2}=1$

These relations (subject to the condition that all $V_{i}, \bar{V}_{i}, E_{j}, \bar{E}_{j}$ should be assigned $\pm \frac{1}{2}$ as a value) are equivalent to

$$
\begin{array}{cc}
V_{1}=V_{2}, & \bar{V}_{1}=\bar{V}_{2}, \\
E_{1}=E_{2}=-E_{3}=-E_{4}, & \bar{E}_{1}=\bar{E}_{2}=-\bar{E}_{3}=-\bar{E}_{4},
\end{array}
$$


(note that the relations $E_{4}=-E_{1}, E_{3}=-E_{2}, \bar{E}_{4}=-\bar{E}_{1}, \bar{E}_{3}=-\bar{E}_{2}$ come from the algebra but they are also geometrically clear). We now claim that

$$
E_{1}+\bar{V}_{1}+\bar{E}_{2}+V_{2}=1
$$

which is proved in Figure 21. Taking into account (2) the last condition is equivalent to

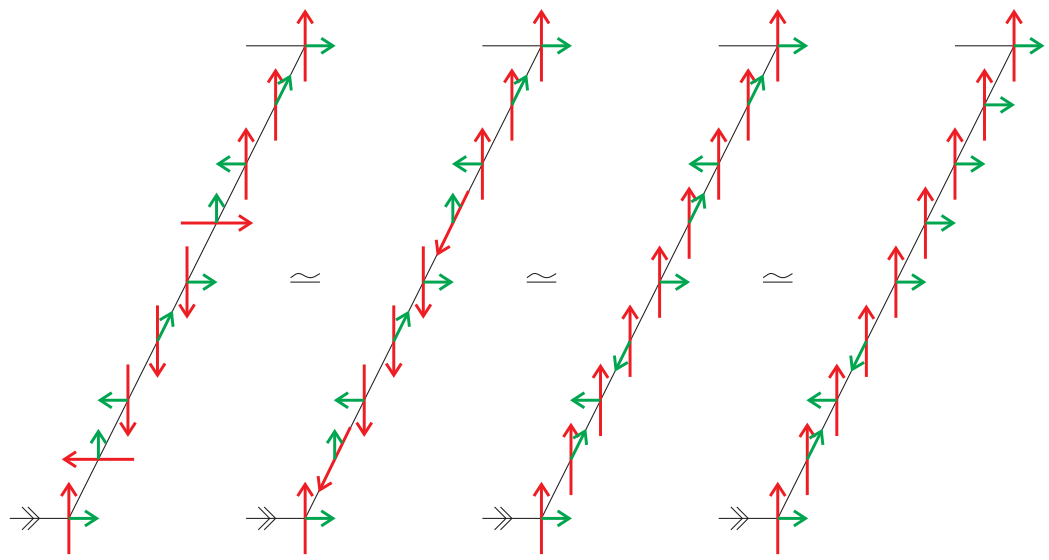

Figure 21: A concatenation giving $1 \in \pi_{1}\left(\mathrm{GL}^{+}(3 ; \mathbb{R})\right)$

any one of the following:

$$
\begin{array}{ll}
V_{1}=E_{1}=\bar{E}_{1}=-\bar{V}_{1}, & V_{1}=E_{1}=\bar{V}_{1}=-\bar{E}_{1}, \\
\bar{V}_{1}=E_{1}=\bar{E}_{1}=-V_{1}, & V_{1}=\bar{E}_{1}=\bar{V}_{1}=-E_{1} .
\end{array}
$$

Choosing one of the relations (3) and combining it with (2) one can now compute the correct value of any possible concatenation according to (1). Let us now choose $V_{1}=E_{1}=\bar{E}_{1}=+\frac{1}{2}$ and $\bar{V}_{1}=-\frac{1}{2}$, and note that the concatenation rules (1) imply that the total number of $V_{1}, V_{2}, \bar{V}_{1}, \bar{V}_{2}$ found along $\partial R$ is even (see also below). The desired computation rule and the last assertion of the statement easily follow.

\subsection{Remarks on the computation of the obstruction}

At the end of the proof of Proposition 2.7 one can also choose $V_{1}=\bar{V}_{1}=E_{1}=+\frac{1}{2}$ and $\bar{E}_{1}=-\frac{1}{2}$, which implies that $\alpha(P, \omega, b)$ can be also computed by decorating the attaching circles of the regions as in Figure 22. More generally, if we indicate by $c_{i}, \bar{c}_{i}$ 

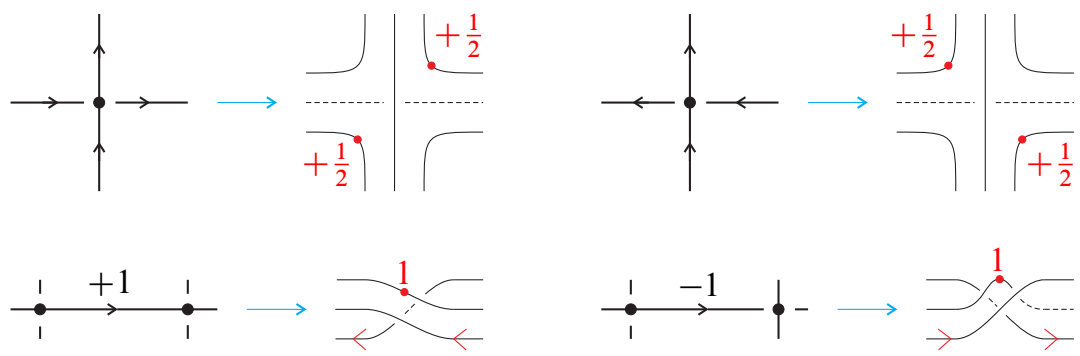

Figure 22: Alternative method to compute $\alpha(P, \omega, b)$

the number of configurations $C_{i}, \bar{C}_{i}$ along $\partial R$, we have that $\alpha_{0}(R)$ is equal to 1 plus

$$
\begin{aligned}
\frac{1}{2}\left(+\left(v_{1}+\right.\right. & \left.\left.v_{2}\right)+\left(\bar{v}_{1}+\bar{v}_{2}\right)+\left(e_{1}+e_{2}-e_{3}-e_{4}\right)-\left(\bar{e}_{1}+\bar{e}_{2}-\bar{e}_{3}-\bar{e}_{4}\right)\right) \\
& =\frac{1}{2}\left(+\left(v_{1}+v_{2}\right)+\left(\bar{v}_{1}+\bar{v}_{2}\right)-\left(e_{1}+e_{2}-e_{3}-e_{4}\right)+\left(\bar{e}_{1}+\bar{e}_{2}-\bar{e}_{3}-\bar{e}_{4}\right)\right) \\
& =\frac{1}{2}\left(+\left(v_{1}+v_{2}\right)-\left(\bar{v}_{1}+\bar{v}_{2}\right)+\left(e_{1}+e_{2}-e_{3}-e_{4}\right)+\left(\bar{e}_{1}+\bar{e}_{2}-\bar{e}_{3}-\bar{e}_{4}\right)\right) \\
& =\frac{1}{2}\left(-\left(v_{1}+v_{2}\right)+\left(\bar{v}_{1}+\bar{v}_{2}\right)+\left(e_{1}+e_{2}-e_{3}-e_{4}\right)+\left(\bar{e}_{1}+\bar{e}_{2}-\bar{e}_{3}-\bar{e}_{4}\right)\right)
\end{aligned}
$$

and these expressions are recognized to be equivalent to each other because

$$
\begin{array}{ll}
v_{1}+v_{2}+\bar{e}_{1}+\bar{e}_{2}+\bar{e}_{3}+\bar{e}_{4}, & v_{1}+v_{2}+e_{1}+e_{2}+e_{3}+e_{4}, \\
\bar{v}_{1}+\bar{v}_{2}+\bar{e}_{1}+\bar{e}_{2}+\bar{e}_{3}+\bar{e}_{4}, & \bar{v}_{1}+\bar{v}_{2}+e_{1}+e_{2}+e_{3}+e_{4},
\end{array}
$$

are all even numbers, thanks to (1). This implies that $v_{1}+v_{2}+\bar{v}_{1}+\bar{v}_{2}$ is also even (as noted above), and $e_{1}+e_{2}+e_{3}+e_{4}+\bar{e}_{1}+\bar{e}_{2}+\bar{e}_{3}+\bar{e}_{4}$ is even as well (which is clear, since it counts the number of up/down switches of $v$ ).

Remark 2.8 The main reason why we have defined $\varphi(P, \omega, b)=(\nu, \mu)$ not as $\left(\nu, \mu_{0}\right)$, but rather adding a full twist to $\mu_{0}$ along unbranched edges, was to have additivity of the frames with respect to edge-fusion, as explained in Proposition 2.6. Coherently with this we now have that the obstruction $\alpha(P, \omega, b)$ is also additive, namely it can be computed at the level of the graphs in $\widetilde{\mathcal{N}}$, which would be false for $\alpha_{0}$. Two examples of additivity (that again holds independently of the interpretation of the 2-valent vertices) are shown in Figure 23.

Remark 2.9 Extending results of [4; 3], in [2] certain quantum hyperbolic invariants $\mathcal{H}_{N}(\mathcal{P})$ have been constructed for a variety of patterns $\mathcal{P}$, with $N \geq 3$ an odd integer. A pattern consists of an oriented compact 3-manifold $M$ with (possibly empty) toric boundary, and an elaborated extra structure on $M$, which includes a PSL(2, $\mathbb{C}$ )character. Each invariant is computed as a state sum over a suitably decorated weakly branched triangulation of $M$ with some number $k$ of punctures, and it is well-defined 


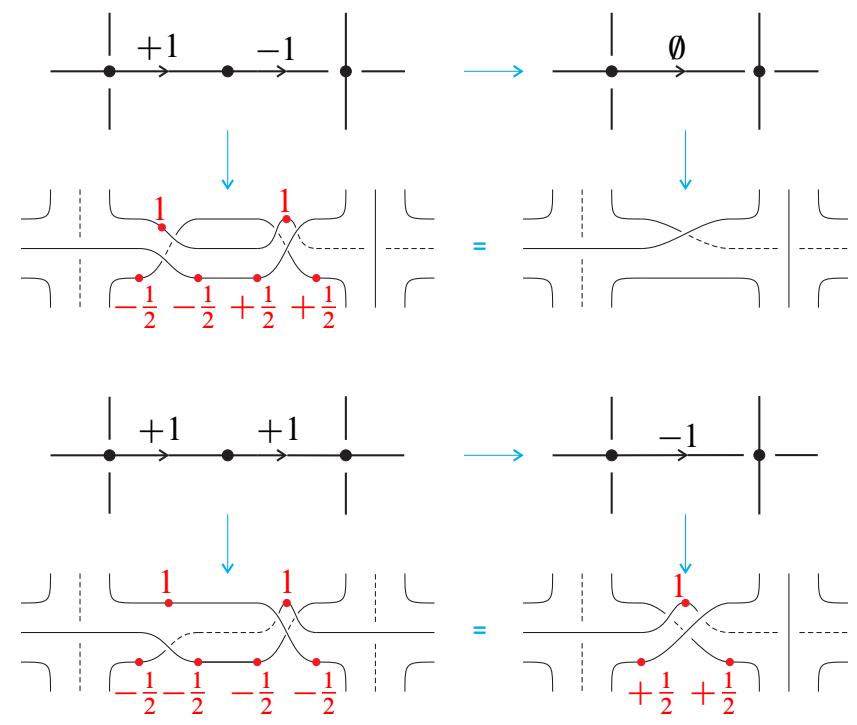

Figure 23: Additivity of the computation of $\alpha$

up to a phase anomaly. Namely, for $N \equiv 1(\bmod 4)$ up to multiplication by an $N^{\text {th }}$ root of unity, while for $N \equiv 3(\bmod 4)$ up to multiplication by an $N^{\text {th }}$ root of unity and a sign. And it turns our that in the latter case the sign ambiguity can be removed by multiplying the state sum by $(-1)^{k-\alpha(P, \omega, b)([P])}$, where $(P, \omega, b)$ is the weakly branched spine dual to the triangulation, and $[P] \in C_{2}(P ; \mathbb{Z} / 2 \mathbb{Z})$ is the sum of all the regions of $P$.

\subsection{Spin structures from cochains}

We close this section with a result that dualizes to Proposition 1.1.

Proposition 2.10 The class of $\alpha=\alpha(P, \omega, b)$ vanishes in $H^{2}(P ; \mathbb{Z} / 2 \mathbb{Z})$. For every $\beta \in C^{1}(P ; \mathbb{Z} / 2 \mathbb{Z})$ such that $\delta \beta=\alpha$ a spin structure $s(P, \omega, b, \beta)$ is well-defined as the homotopy class of the frame $(\nu, \beta(\mu))$ on $S(P)$, where $(\nu, \mu)=\varphi(P, \omega, b)$ and $\beta(\mu)$ is obtained by giving a full twist to $\mu$ along all the edges $e$ of $P$ such that $\beta(e)=1$. Moreover $s\left(P, \omega, b, \beta_{0}\right)=s\left(P, \omega, b, \beta_{1}\right)$ if and only if $\beta_{0}+\beta_{1}$ vanishes in $H^{1}(P ; \mathbb{Z} / 2 \mathbb{Z})$.

Proof All three assertions are general topological facts. To prove the first one, let $(\bar{\nu}, \bar{\mu})$ be any given spin structure on $M$, namely a frame on $S(P)$ that extends to $P$ and is viewed up to homotopy on $S(P)$. Homotoping $(\bar{\nu}, \bar{\mu})$ we can suppose it coincides with $(\nu, \mu)$ at the vertices of $P$, so we can define $\beta \in C^{1}(P ; \mathbb{Z} / 2 \mathbb{Z})$ where $\beta(e)$ is 
the difference between $(v, \mu)$ and $(\bar{v}, \bar{\mu})$ along $e$. Since the obstruction to extending $(\bar{v}, \bar{\mu})$ to a region $R$ of $P$ vanishes, we see that the obstruction $\alpha(R)$ to extending $(\nu, \mu)$ to $R$ is the sum of $\beta(e)$ for all the edges $e$ of $P$ contained in $\partial R$, namely $\delta \beta=\alpha$.

The second assertion is now easy: if $\delta \beta=\alpha$ then the obstruction to extending $(\nu, \beta(\mu))$ to $P$ vanishes.

Turning to the third assertion, it is first of all evident that if $v$ is a vertex of $P$ and $\hat{v} \in C^{0}(P ; \mathbb{Z} / 2 \mathbb{Z})$ is its dual then the frames on $S(P)$ carried by some $\beta$ with $\delta \beta=\alpha$ and by $\beta+\delta \hat{v}$ are homotopic on $S(P)$, with homotopy supported near $v$. Conversely, suppose $\beta_{0}, \beta_{1}$ with $\delta \beta_{0}=\delta \beta_{1}=\alpha$ give frames $\left(v^{(0)}, \mu^{(0)}\right)$ and $\left(v^{(1)}, \mu^{(1)}\right)$ that are homotopic on $S(P)$ via $\left(v^{(t)}, \mu^{(t)}\right)_{t \in[0,1]}$. If $v$ is a vertex of $P$, by construction $\left(v^{(0)}, \mu^{(0)}\right)$ equals $\left(v^{(1)}, \mu^{(1)}\right)$ at $v$, so we can view $\left(v^{(t)}, \mu^{(t)}\right)_{t \in[0,1]}$ at $v$ as an element $\gamma(v)$ of $\pi_{1}\left(\mathrm{GL}^{+}(3 ; \mathbb{R})\right)=\mathbb{Z} / 2 \mathbb{Z}$. We then have $\gamma \in C^{0}(P ; \mathbb{Z} / 2 \mathbb{Z})$ and $\beta_{1}=\beta_{0}+\delta \gamma$, whence the conclusion.

Note that the previous result is coherent with the known fact that the set of spin structures on $M$ is an affine space over $H^{1}(P ; \mathbb{Z} / 2 \mathbb{Z})=H^{1}(M ; \mathbb{Z} / 2 \mathbb{Z})$.

\section{Spine moves preserving the spin structure}

We will establish in this section the dual versions of Propositions 1.3 to 1.5. From now on we will regard any $\beta \in C^{1}(P ; \mathbb{Z} / 2 \mathbb{Z})$ such that $\delta \beta=\alpha(P, \omega, b)$ up to coboundaries. To discuss when two quadruples $(P, \omega, b, \beta)$ define the same $s(P, \omega, b, \beta)$ we can then describe right to left how the quadruple must change, and we have already dealt with the change of $\beta$.

Before proceeding further we introduce a convenient graphic encoding for the quadruples $(P, \omega, b, \beta)$. Namely we define $\mathcal{N}_{\mathrm{w}}$ as the set of all graphs $\Gamma$ as in $\mathcal{N}$, with the extra structure of a weight in $\mathbb{Z} / 2 \mathbb{Z}$ attached to each edge of $\Gamma$. A natural correspondence between $\mathcal{N}_{\mathrm{w}}$ and the set of all quadruples $(P, \omega, b, \beta)$, with $(P, \omega, b)$ as in Proposition 2.4 and $\beta \in C^{1}(P ; \mathbb{Z} / 2 \mathbb{Z})$, is obtained by interpreting the weight of an edge as the value of $\beta$ on it. Note that for $\Gamma \in \mathcal{N}_{\mathrm{w}}$ the edge colors belong to $\mathbb{Z} / 3 \mathbb{Z}=\{\emptyset,+1,-1\}$ and the weights to $\mathbb{Z} / 2 \mathbb{Z}=\{0,1\}$, so no confusion between colors and weights is possible. Colors $\emptyset$ and weights 0 will often be omitted. We can similarly define $\widetilde{\mathcal{N}}_{\mathrm{w}}$ as the set of graphs in $\widetilde{\mathcal{N}}$ with weights in $\mathbb{Z} / 2 \mathbb{Z}$ attached to the edges, stipulating that weights sum up in $\mathbb{Z} / 2 \mathbb{Z}$ when two edges are fused together. 


\subsection{The vertex moves}

The next result dualizes to Proposition 1.3.

Proposition 3.1 Two graphs in $\mathcal{N}_{\mathrm{w}}$ define quadruples $\left(P_{j}, \omega_{j}, b_{j}, \beta_{j}\right)$ for $j=0,1$ with $P_{1}=P_{0}, \omega_{1}=\omega_{0}$ and $s\left(P_{0}, \omega_{0}, b_{0}, \beta_{0}\right)=s\left(P_{1}, \omega_{1}, b_{1}, \beta_{1}\right)$ if and only if they are obtained from each other by repeated applications of the moves $I$ and II of Figure 24 (and their inverses, followed by the reduction from $\widetilde{\mathcal{N}}$ to $\mathcal{N}$ ).
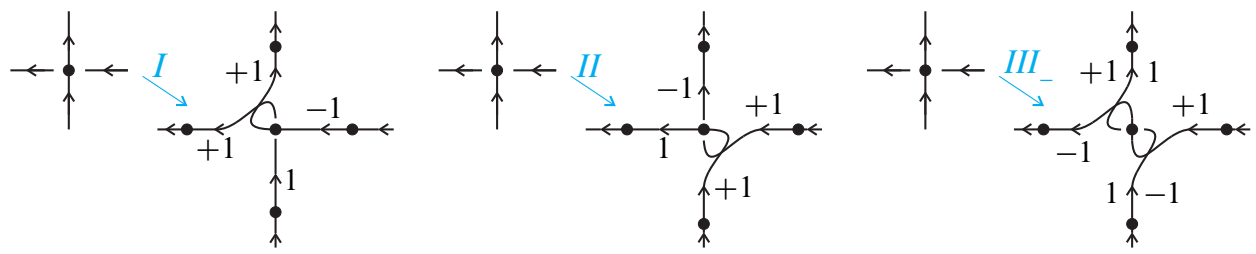

Figure 24: Moves that change the weak branching while preserving the prebranching and the spin structure. Recall that \pm 1 are colors in $\mathbb{Z} / 3 \mathbb{Z}$ while 1 is a weight in $\mathbb{Z} / 2 \mathbb{Z}$.

Proof We must prove that the moves $I$ and $I I$ generate all possible changes at a vertex $V$ of a weak branching compatible with a given prebranching and, taking weights into account, that the associated spin structure is preserved. For both indices $\varepsilon= \pm 1$ of $V$ there are 3 such possible changes; for $\varepsilon=-1$ they are given by the moves $I, I I$ and $I I I_{-}$(already shown in Figure 24), which can be realized as $I I I_{-}=I \cdot \overline{I I}=I I \cdot \bar{I}$, with $\bar{I}$ and $\bar{I}$ the inverses of $I$ and $I I$, and products written with the move applied first on the left; for $\varepsilon=+1$ the 3 possible changes are given by $\bar{I}, \overline{I I}$ and $I I I+=\overline{I I} \cdot I=\bar{I} \cdot I I$. It is then sufficient to show that the moves $I$ and $I I$ correctly represent one change of weak branching and preserve the spin structure, which we will do explicitly only for $I$. Ignoring the frame, the proof that $I$ preserves the prebranched spine is contained in Figure 25(left). Turning to the frames, thanks to Proposition 2.6, we can carry out a completely local analysis. Moreover we note that locally before the move the frame $(\nu, \beta(\mu))$ coincides with $\left(\nu, \mu_{0}\right)$, while after the move the frame $(\nu, \beta(\mu))$ is obtained from $\left(\nu, \mu_{0}\right)$ by giving a full twist to $\mu_{0}$ along each of the 4 involved edges (three edges have color \pm 1 and weight 0 , the fourth edge has color $\emptyset$ and weight 1$)$. These four twists are induced by a homotopy, so it will be enough to show that the frames $\left(v, \mu_{0}\right)$ before and after the move coincide up to homotopy. Showing this on a single global picture is too complicated, so we confine ourselves to proving that $\left(\nu, \mu_{0}\right)$ is unchanged up to homotopy separately on the boundary of each of the regions $A, B, C, D, E, F$ of Figure 25(left). This is very easy for all the regions except $A$; see for instance Figure 25(right) for $D$. In Figure 26 we treat instead the case of the region $A$. 

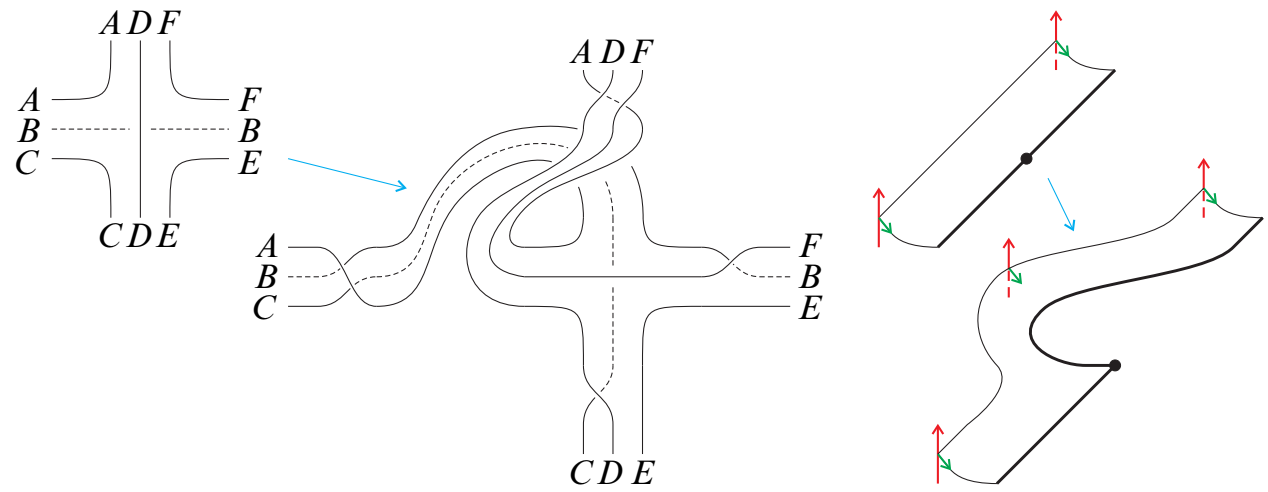

Figure 25: Left: move $I$ preserves the prebranched spine Right: the frame $\left(\nu, \mu_{0}\right)$ is unchanged under move $I$ on the region $D$
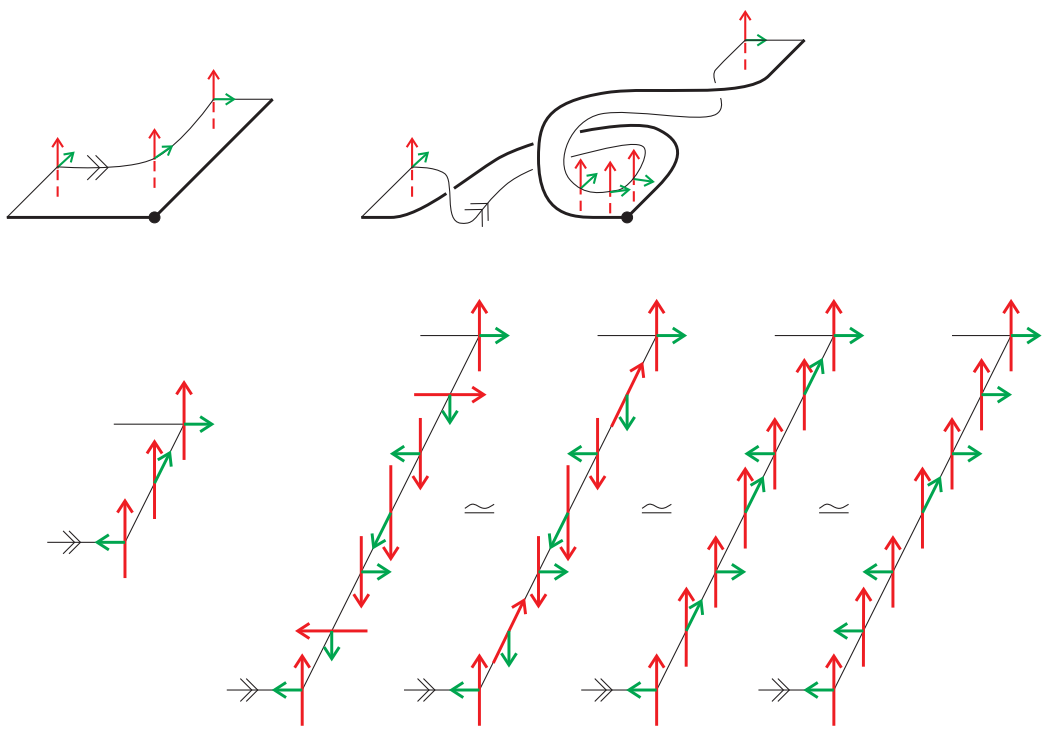

Figure 26: The frame $\left(v, \mu_{0}\right)$ is unchanged up to homotopy on $A$. Left: before the move Right: after the move Top: locally embedded configuration Bottom: abstract configuration

Remark 3.2 Let the change of weak branching on the prebranched spine $(P, \omega)$ in move $I$ be given by $b \mapsto b^{\prime}$. The difference $\Delta \alpha=\alpha(P, \omega, b)+\alpha\left(P, \omega, b^{\prime}\right)$ is then computed locally, and Proposition 3.1 implies that $\Delta \alpha=\delta \widehat{e}$, with $e$ as in Figure 27. This fact can actually be checked directly, as in the rest of Figure 27, since the picture shows that $\Delta \alpha$ is 0 on $A, B, F$ and 1 on $C, D, E$. 


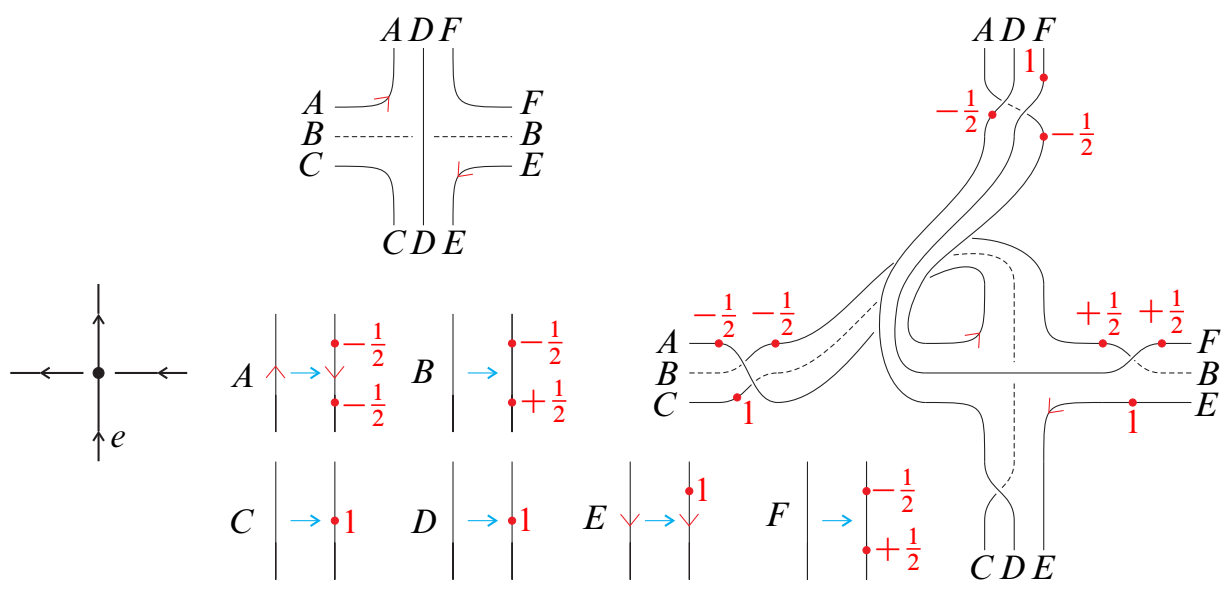

Figure 27: Variation of $\alpha$ with move $I$

\subsection{The circuit move}

The next result dualizes to Proposition 1.4.

Proposition 3.3 Two graphs in $\mathcal{N}_{\mathrm{w}}$ define quadruples $\left(P_{j}, \omega_{j}, b_{j}, \beta_{j}\right)$ for $j=0,1$ with $P_{1}=P_{0}$ and $s\left(P_{0}, \omega_{0}, b_{0}, \beta_{0}\right)=s\left(P_{1}, \omega_{1}, b_{1}, \beta_{1}\right)$ if and only if they are related by the moves of Proposition 3.1 plus moves of the form $\Gamma \mapsto \Gamma^{\prime}$, where

- $\quad \Gamma$ contains a simple oriented circuit $\gamma$ that at all its vertices is an overarc,

- $\Gamma^{\prime}$ is obtained from $\Gamma$ by reversing the orientation of the edges in $\gamma$ and adding 1 to the weights of the edges of $\gamma$ whose ends have distinct indices.

Proof Suppose that $\omega_{0}$ and $\omega_{1}$ are distinct prebranchings on the same spine $P$. The union of the edges of $P$ on which $\omega_{0}$ and $\omega_{1}$ disagree can be expressed as a disjoint union of simple circuits oriented by $\omega_{0}$. It is then sufficient to consider the situation of two weak branchings $\omega, \omega^{\prime}$ that differ only on a simple circuit $\gamma$ oriented by $\omega$, and then iterate the procedure. Moreover, having already described how to obtain from each other any two pairs $(b, \beta)$ yielding the same spin structure on a given $(P, \omega)$, it is now sufficient to find one specific weak branching $b$ on $(P, \omega)$ and one $b^{\prime}$ on $\left(P, \omega^{\prime}\right)$ and to describe a move $\beta \mapsto \beta^{\prime}$ such that $s(P, \omega, b, \beta)=s\left(P, \omega^{\prime}, b^{\prime}, \beta^{\prime}\right)$. This move will be that of the statement, implying the conclusion. To describe the move we note that indeed via Proposition 3.1 we can arrange so that $\gamma$ contains overarcs only in a graph $\Gamma \in \mathcal{N}$ giving a weak branching $b$ on $(P, \omega)$. Examining Figures 1 (left) and 13 one readily sees that the weak branching $b^{\prime}$ obtained by reversing $\gamma$ is derived from $b$ by switching the orientation of the edge $v_{2} v_{3}$ in the tetrahedra dual to the edges in $\gamma$. We 
are only left to show that the move $\beta \mapsto \beta^{\prime}$ such that $s(P, \omega, b, \beta)=s\left(P, \omega^{\prime}, b^{\prime}, \beta^{\prime}\right)$ consists in adding to $\beta$ the 1-cochain $\Delta \beta$ given by the duals of the edges in $\gamma$ having endpoints with distinct indices. We will prove this in a slightly indirect way, in the spirit of Remark 3.2, by computing $\Delta \alpha=\alpha(P, \omega, b)+\alpha\left(P, \omega^{\prime}, b^{\prime}\right)$ and showing that $\Delta \alpha=\delta(\Delta \beta)$.
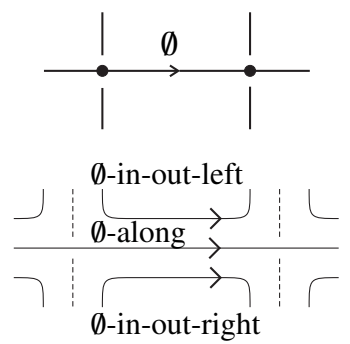
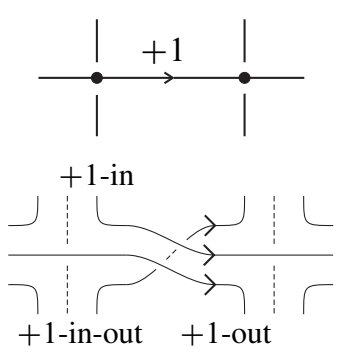
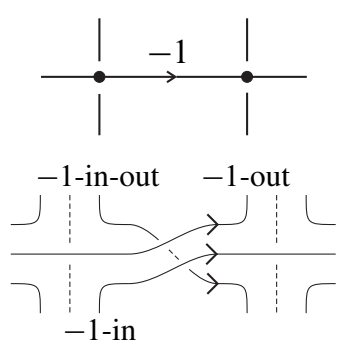

Figure 28: The edges in the circuit $\gamma$ and the corresponding regions of $P$

We begin by noting that there are nine possible positions of a region $R$ with respect to an edge $e$ of $\gamma$, as shown in Figure 28. One can now check that the contributions carried by $e$ to $(\Delta \alpha)(R)$, depending on the indices $\pm 1 / \pm 1$ of the ends of $e$, are as given in the following table (with $\partial R$ oriented as in Figure 28):

\begin{tabular}{c||c|c|c|c} 
& $+1 /+1$ & $-1 /-1$ & $+1 /-1$ & $-1 /+1$ \\
\hline \hline$\emptyset$-in-out-right & 0 & 0 & 1 & 1 \\
\hline$\emptyset$-along & 0 & 0 & 0 & 0 \\
\hline$\emptyset$-in-out-left & 0 & 0 & 1 & 1 \\
\hline+1 -in-out & 0 & 0 & 1 & 1 \\
\hline+1 -in & 1 & 0 & 1 & 0 \\
\hline+1 -out & 1 & 0 & 0 & 1 \\
\hline-1 -in-out & 0 & 0 & 1 & 1 \\
\hline-1 -in & 1 & 0 & 1 & 0 \\
\hline-1 -out & 1 & 0 & 0 & 1
\end{tabular}

See Figure 29 for the explicit computation of some of these values.

To conclude we must now show that the total $(\Delta \alpha)(R)$ obtained by summing the contributions given by the various edges of $\gamma$ equals $(\bmod 2)$ the number of edges in $\gamma$ visited by $\partial R$ and having ends with distinct indices. If $\partial R$ visits only one edge, $i e$, if it is of type in-out, the conclusion is evident from the values in the table. Otherwise $(\Delta \alpha)(R)$ is the sum of only two possibly nonzero contributions, one from the edge of $\gamma$, where $\partial R$ enters and one from the edge of $\gamma$ where it leaves. More precisely, as 


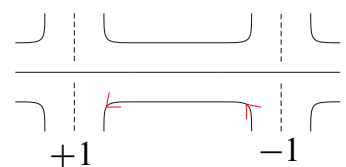

$\emptyset$
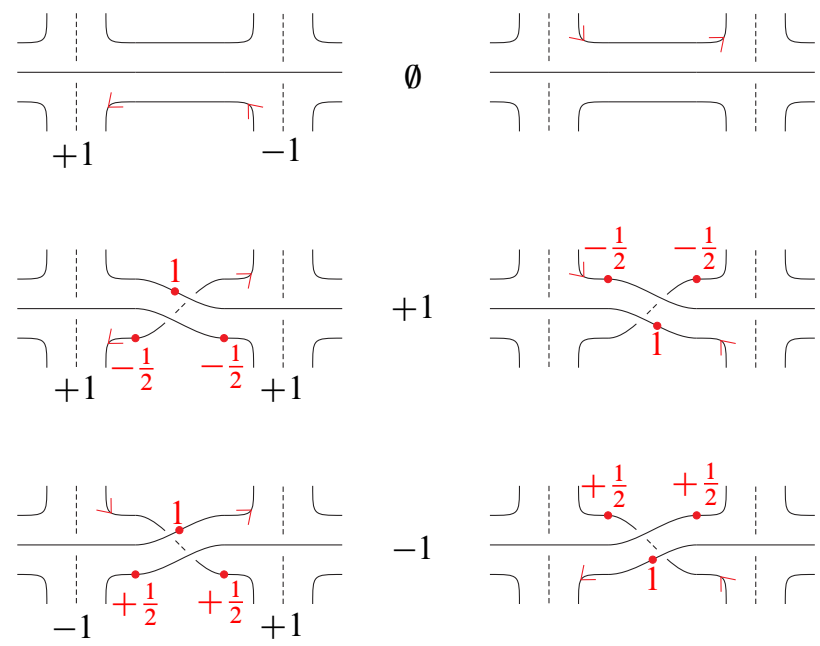

Figure 29: Three computations of the contribution of an edge $e$ in $\gamma$ to $(\Delta \alpha)(R)$. The color $\emptyset /+1 /-1$ of $e$ (unchanged by the switch of $\gamma$ ) is shown in the middle. On the left we see the indices of the ends of $e$ before the switch, and the local computation of $\alpha(R)$; on the right the computation of $\alpha^{\prime}(R)$ after the switch.

one sees from the table, there is an "in" contribution depending only on the index of the vertex of $\gamma$ where $\partial R$ enters (contribution 1 for index +1 and contribution 0 for index -1 ), and an "out" contribution depending only on the index of the vertex of $\gamma$ where $\partial R$ leaves (again, contribution 1 for index +1 and contribution 0 for index -1 ). This implies that indeed $(\Delta \alpha)(R)$ has the desired value, and the proof is complete.

\subsection{The bubble and the $2-3$ move}

The next result dualizes to Proposition 1.5.

Proposition 3.4 Two graphs in $\mathcal{N}_{\mathrm{w}}$ define quadruples $\left(P_{j}, \omega_{j}, b_{j}, \beta_{j}\right)$ for $j=0,1$ with $s\left(P_{0}, \omega_{0}, b_{0}, \beta_{0}\right)=s\left(P_{1}, \omega_{1}, b_{1}, \beta_{1}\right)$ if and only if they are obtained from each other by the moves of Propositions 3.1 and 3.3 and those shown in Figure 30.

Proof Two special polyhedra are spines (in the punctured sense) of the same manifold without boundary spheres if and only if they are related by bubble and 2-3 moves; see Matveev [15] and Piergallini [17]. It is then sufficient to prove the following:

- using the moves $I$ and $I I$ any edge $e$ with distinct ends $V_{0}, V_{1}$ of a graph in $\mathcal{N}_{\mathrm{w}}$ can be transformed into one to which a move in Figure 30 applies; 

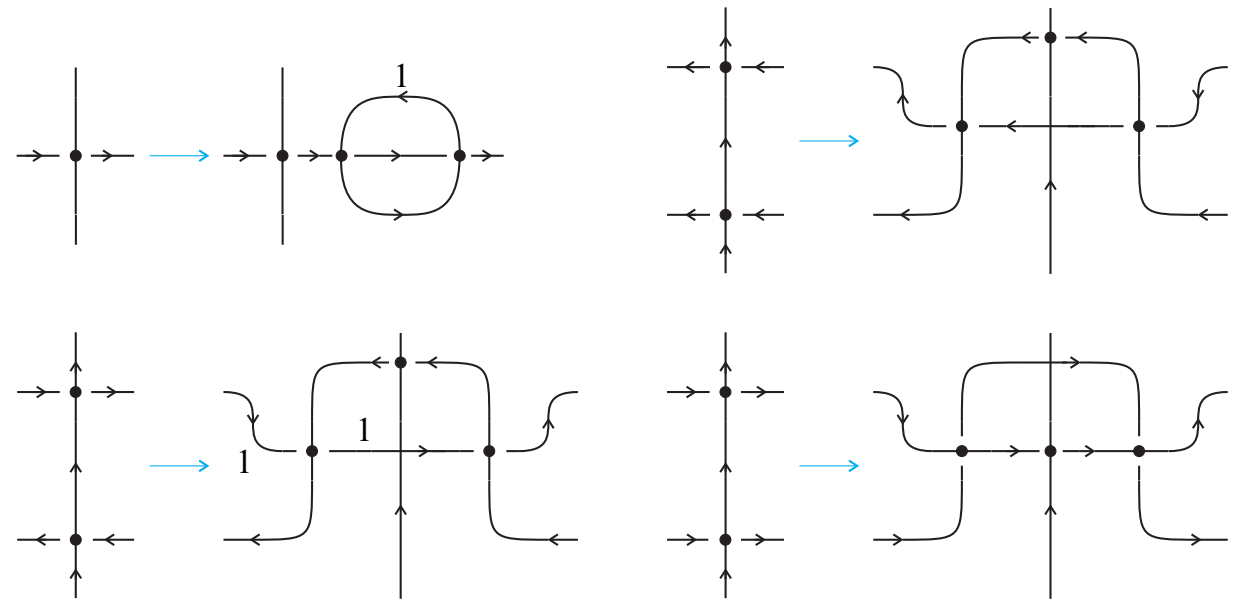

Figure 30: Moves on $\widetilde{\mathcal{N}}_{\mathrm{w}}$ preserving the associated spin structure; the edges entirely contained in the picture have color $\emptyset$ and weight 0 .

- at the level of spines the moves in Figure 30 translate the bubble and the 2-3 move, and at the level of quadruples $(P, \omega, b, \beta)$ represented by graphs in $\mathcal{N}_{\mathrm{w}}$ the associated spin structure is unchanged under these moves.

With $\varepsilon$ being the index of a vertex, the following steps establish the first assertion:

(1) if $e$ is an underpass at some $V_{j}$, apply to each such $V_{j}$ the move $I I I_{\varepsilon\left(V_{j}\right)}$ (with $I I I_{+}$the analogue of $I I I_{-}$for a vertex of index +1 ); this allows us to assume that $e$ is an overpass at $V_{0}$ and $V_{1}$;

(2) if the color of $e$ is now +1 , act as follows:

(a) if $\varepsilon\left(V_{0}\right)=\varepsilon\left(V_{1}\right)=-1$, apply $I$ to $V_{0}$ and $I I$ to $V_{1}$;

(b) if $\varepsilon\left(V_{0}\right)=-1$ and $\varepsilon\left(V_{1}\right)=+1$, apply $\overline{I I}$ to $V_{1}$;

(c) if $\varepsilon\left(V_{0}\right)=+1$, apply $\bar{I}$ to $V_{0}$;

(3) if the color of $e$ is now -1 , act as follows:

(a) if $\varepsilon\left(V_{0}\right)=\varepsilon\left(V_{1}\right)=+1$, apply $\bar{I}$ to $V_{0}$ and $\overline{I I}$ to $V_{1}$;

(b) if $\varepsilon\left(V_{0}\right)=+1$ and $\varepsilon\left(V_{1}\right)=-1$, apply $I I$ to $V_{1}$;

(c) if $\varepsilon\left(V_{0}\right)=-1$, apply $I$ to $V_{0}$;

(4) the color of $e$ is now $\emptyset$, and we want to exclude the case $\varepsilon\left(V_{0}\right)=+1$ and $\varepsilon\left(V_{1}\right)=-1$, for which we apply $\bar{I}$ to $V_{0}$ and $I I$ to $V_{1}$;

(5) up to coboundaries we turn the weight of $e$ to 0. 

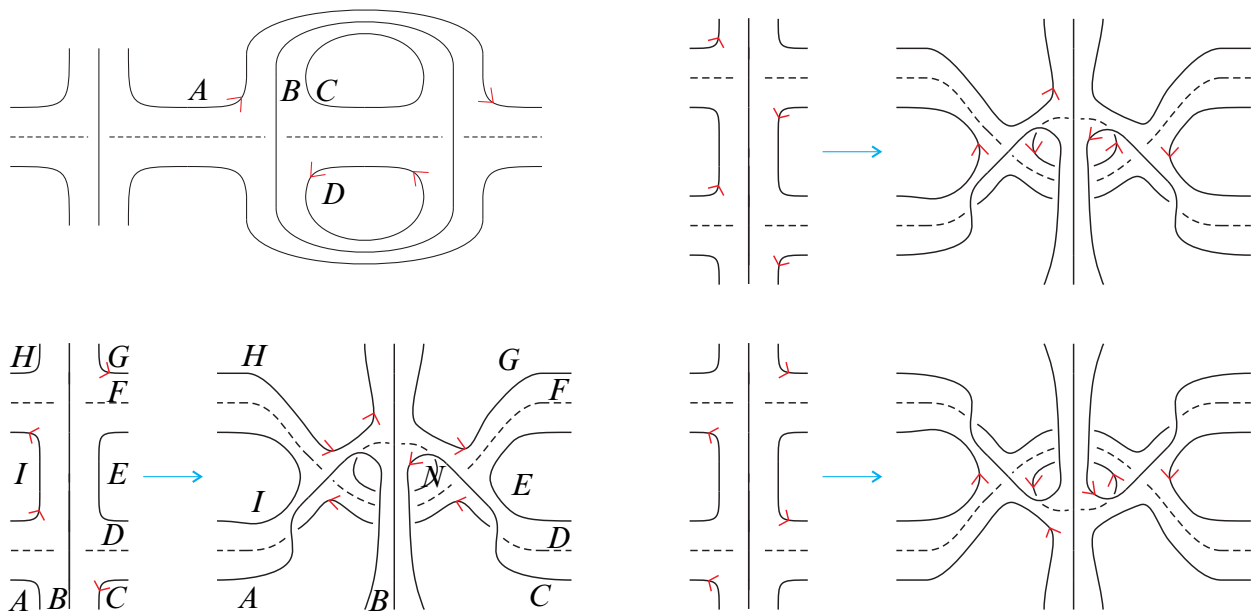

Figure 31: Proof that under the moves of Figure 30 the change in the obstruction $\alpha$ is compensated by the weights on the edges. For the two moves on the right this is easy: on all the regions that survive $\alpha$ keeps the same value, and on the newborn region it has value 0 . For the top left move $\Delta \alpha$ is 1 on $A, B, C$ and 0 on $D$, while for the bottom left move $\Delta \alpha$ is 0 on $A, B, C, E, F, G$ and 1 on $D, H, I, N$, and indeed for both cases these values are given by the weights in the moves.

For the second assertion, once again we start by an indirect argument in the spirit of Remark 3.2, showing that the weights appearing in the moves compensate for the variation of the obstruction $\alpha \in C^{2}(P ; \mathbb{Z} / 2 \mathbb{Z})$, which is done in Figure 31. A more direct argument for the move of Figure 30(top/left) is carried out in Figure 32; for the other moves the argument follows from [6].

\section{Arbitrarily branched graphs and the corresponding moves}

In this section we show that the global move of Proposition 3.3 can be replaced, in a suitable sense, by a simultaneous combination of local ones.

\subsection{Graphs representing arbitrarily branched triangulations}

We introduce now a set $\mathcal{A}$ of decorated graphs via which we can encode an arbitrarily branched triangulation, namely a triangulation in which each tetrahedron is endowed with a branching, without any compatibility whatsoever. Each vertex of a graph $\Gamma$ in $\mathcal{A}$ will be given a planar structure as in Figure 13, which corresponds to giving the dual tetrahedron a branching. Note that each edge of $\Gamma$ then has an orientation defined at 

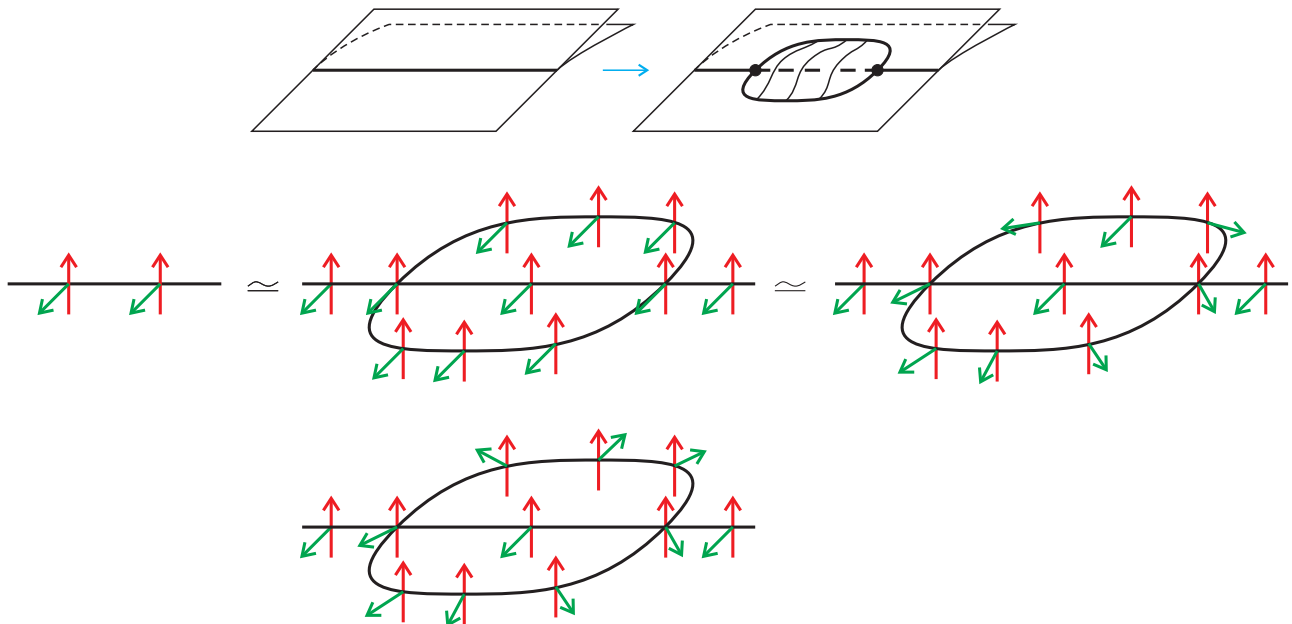

Figure 32: Top: the branched bubble move. Middle: frames carrying the same spin structure before and after the move. Bottom: the frame carried by the spine after the move, that becomes the previous one taking into account the weight 1 appearing in the move of Figure 30(left).

each of its ends. We are left to choose colors for the edges of $\Gamma$ in order to encode the face pairings, or equivalently the attaching circles to $S(P)=\Gamma$ of the regions of the dual spine $P$. To do so we note that dual to a germ $e$ of edge of $S(P)$ at some vertex there is a branched triangle. We can now label by $0,1,2$ the vertices of this triangle according to the number of incoming edges, and dually the germs of regions incident to $e$. We show in Figure 33(left) (in a cross section) this abstract labeling rule, and in Figure 33(right) its concrete consequences. One can now easily check the following.
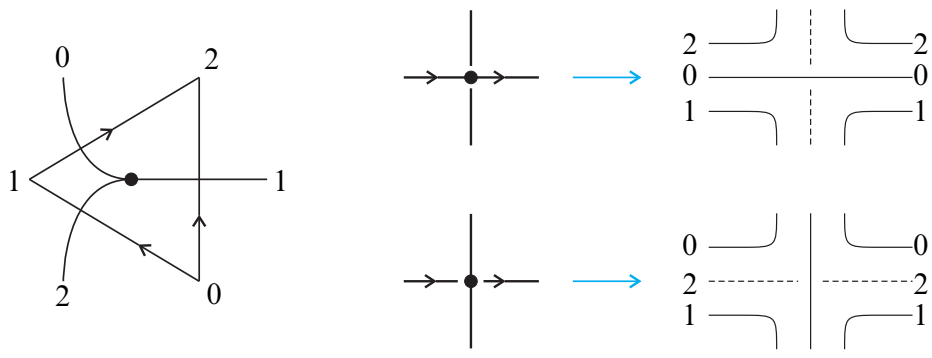

Figure 33: Labels for the germs of region near a (branched) vertex

Lemma 4.1 Let $e$ be an edge of $\Gamma$ and let $n(e)$ be the number of regions incident to $e$ having the same label at both ends of $e$. 
- If the two ends of $e$ are consistently oriented then $n(e)=0$ or $n(e)=3$.

- If the two ends of $e$ are inconsistently oriented then $n(e)=1$.

This implies that we can give an edge $e$ of $\Gamma$ the following colors in $\mathfrak{S}_{3}$ (see Figure 34 for some examples):

- if the two ends of $e$ are consistently oriented, color

$$
\sigma \in \mathfrak{S}_{3}^{+}=\left\{\emptyset,\left(\begin{array}{lll}
0 & 1 & 2
\end{array}\right),\left(\begin{array}{lll}
0 & 2 & 1
\end{array}\right)\right\}
$$

if region $j$ at the first end of $e$ is matched to region $\sigma(j)$ at the second end;

- if the two ends of $e$ are inconsistently oriented, color

$$
\tau \in \mathfrak{S}_{3}^{-}=\left\{\left(\begin{array}{ll}
0 & 1
\end{array}\right),\left(\begin{array}{ll}
0 & 2
\end{array}\right),\left(\begin{array}{ll}
1 & 2
\end{array}\right)\right\}
$$

if region $j$ at one end is matched to region $\tau(j)$ at the other end.
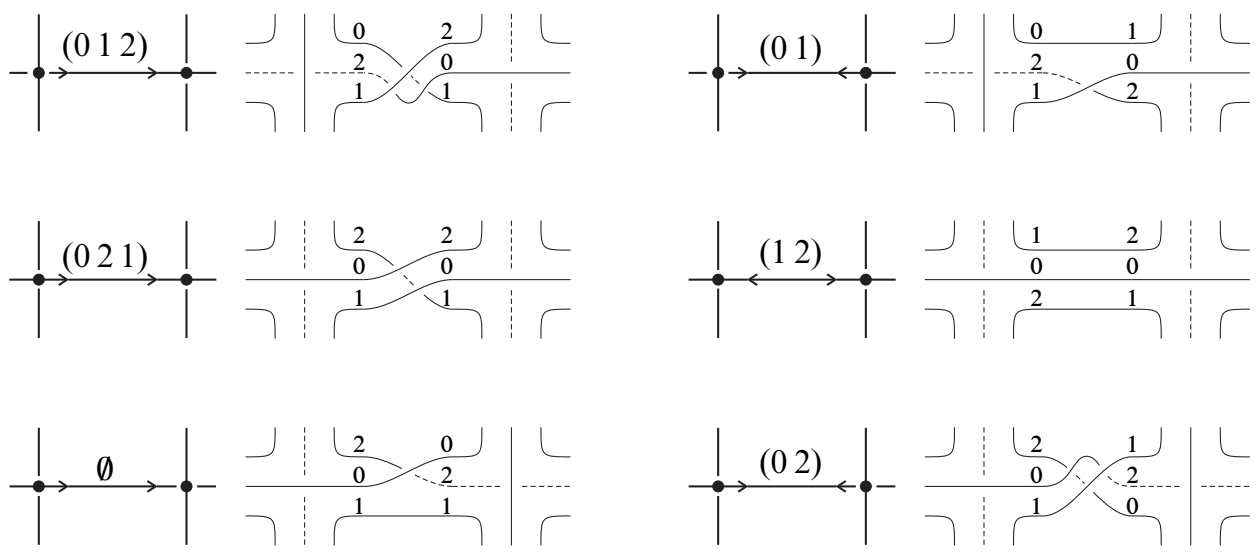

Figure 34: Meaning of the edge colors for a graph in $\mathcal{A}$

Remark 4.2 A graph $\Gamma$ in $\mathcal{A}$ defines a weakly branched triangulation if and only if all the edges are consistently oriented. In this case $\Gamma$ is converted into a graph in $\mathcal{N}$ representing the same weakly branched triangulation by the color-replacements $\left(\begin{array}{lll}0 & 1 & 2\end{array}\right) \mapsto+1$ and $\left(\begin{array}{llll}0 & 2 & 1\end{array}\right) \mapsto-1$.

From now on we will call even (respectively, odd) an edge of a graph in $\mathcal{A}$ with color in $\mathfrak{S}_{3}^{+}$(respectively, in $\mathfrak{S}_{3}^{-}$), or, equivalently, with consistently (respectively, inconsistently) oriented ends. 


\subsection{Graphs with multiply colored edges}

As we did for $\mathcal{N}$, to define moves on $\mathcal{A}$ it is convenient to enlarge it to some $\widetilde{\mathcal{A}}$ by allowing valence- 2 vertices; edges are again decorated by an orientation at each of their ends and a color (in $\mathfrak{S}_{3}^{+}$if the orientations match, in $\mathfrak{S}_{3}^{-}$if they do not), but we also insist that orientations should match across the valence- 2 vertices. Note that if we choose for each $2-$ valent vertex of $\widetilde{\Gamma} \in \widetilde{\mathcal{A}}$ an interpretation as $\equiv$ or as $\overline{\overline{-z}}$ we can associate to $\widetilde{\Gamma}$ an arbitrarily branched special spine.
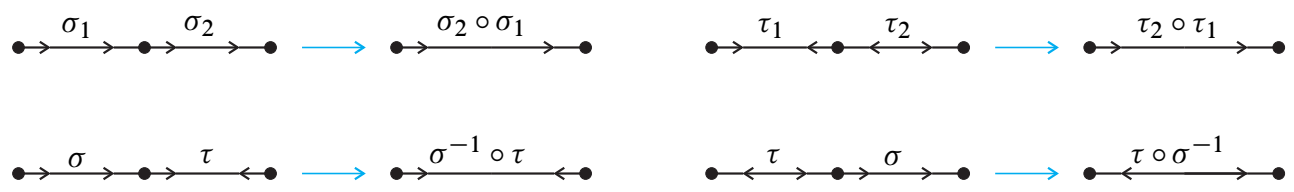

Figure 35: How to fuse together two edges of a graph in $\widetilde{\mathcal{A}}$

We now define a projection $\widetilde{\mathcal{A}} \rightarrow \mathcal{A}$ by illustrating in Figure 35 how fuse together two edges sharing a valence- 2 vertex; note that $\sigma, \sigma_{1}, \sigma_{2} \in \mathfrak{S}_{3}^{+}$and $\tau, \tau_{1}, \tau_{2} \in \mathfrak{S}_{3}^{-}$; moreover $\sigma^{-1} \circ \tau=\tau \circ \sigma$ and $\tau \circ \sigma^{-1}=\sigma \circ \tau$, which gives alternative ways of expressing the fusion rules. We have the following.

Proposition 4.3 The fusion rules of Figure 35 are associative, so each graph $\widetilde{\Gamma} \in \widetilde{\mathcal{A}}$ defines a unique $\Gamma \in \mathcal{A}$. Moreover the arbitrarily branched spine associated to $\widetilde{\Gamma}$ is well-defined regardless of the interpretation of the valence- 2 vertices as $\equiv$ or $\overline{-\overline{-}}$, and it coincides with the arbitrarily branched spine associated to $\Gamma$.

The first assertion of this result follows from the second one, that can be established with some patience; see some examples in Figure 36.

\subsection{A new move}

Let us consider the move on graphs in $\widetilde{\mathcal{A}}$ described in Figure 37(left). In Figure 37(right) we show that the move preserves the spine (or triangulation) encoded by the graph, while of course changing the arbitrary branching. The following result (that will also follow from the rest of this section) is not difficult to show:

Proposition 4.4 Any two arbitrary branchings on the same triangulations are related by compositions of the moves I and II (ignoring weights), that of Figure 37, and their inverses.

Since for a single tetrahedron there are 24 different branchings, this result means that at each vertex using the moves $I$ and $I I$ and that of Figure 37 one can create all 24 possible configurations; see for instance Figure 38. 

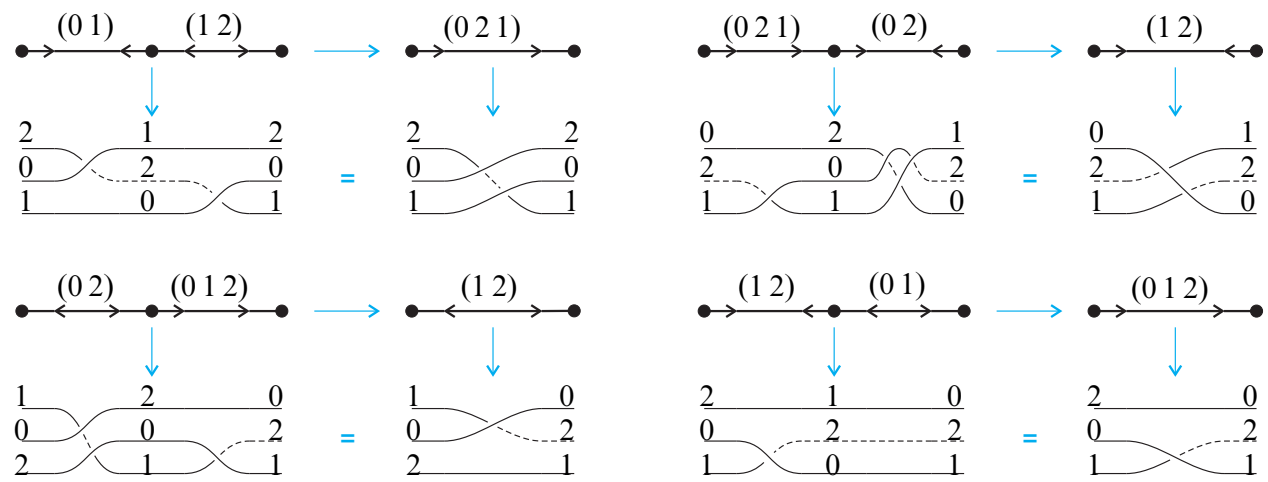

Figure 36: To each $\widetilde{\Gamma} \in \widetilde{\mathcal{A}}$ one can uniquely associate an arbitrarily branched special spine, also given by the graph $\Gamma \in \mathcal{A}$ obtained from $\widetilde{\Gamma}$ by fusing the edges through valence- 2 vertices.
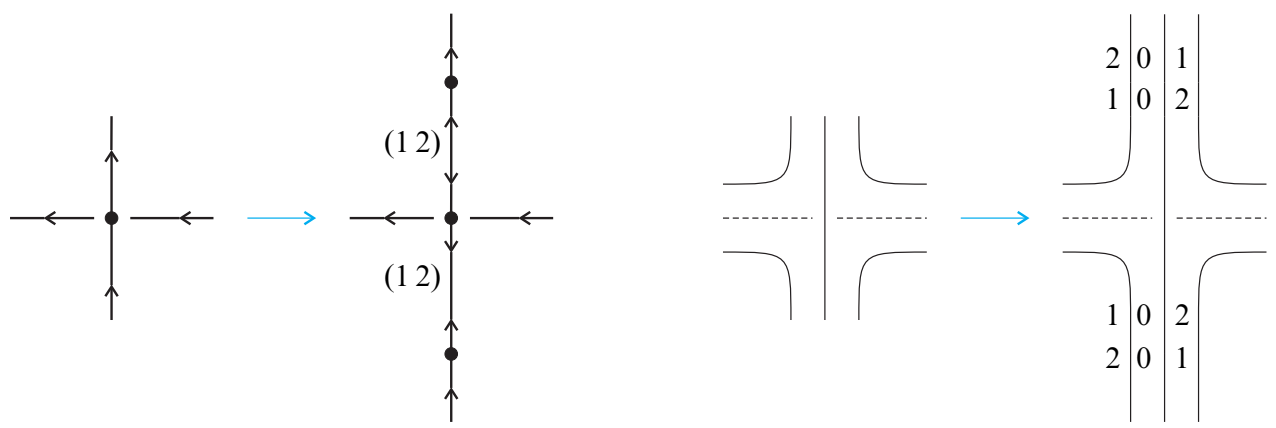

Figure 37: A move on $\tilde{\mathcal{A}}$
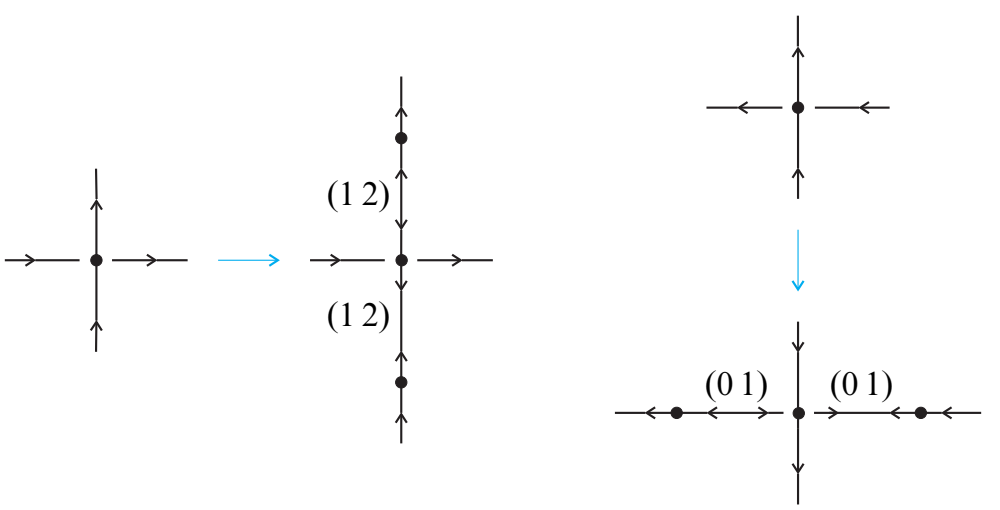

Figure 38: The inverse of the move of Figure 37 and one generated by those in Figures 37 and 24 


\subsection{Weighted graphs and weighted fusion}

We define $\widetilde{\mathcal{A}}_{\mathrm{w}}$ as the set of graphs in $\widetilde{\mathcal{A}}$ with weights attached to the edges. The weight of an edge $e$ is given by an internal orientation and by a numerical weight in the group $G=\left(\frac{1}{2} \cdot \mathbb{Z}\right) / 2 \mathbb{Z}$, with the following restrictions:

- if $e$ is even then its internal orientation matches those at its ends (so it is not shown in the pictures) and the numerical weight is 0 or 1 ;

- if $e$ is odd the numerical weight is $\pm \frac{1}{2}$.

Note that there is a natural inclusion $\widetilde{\mathcal{N}}_{\mathrm{w}} \subset \widetilde{\mathcal{A}}_{\mathrm{w}}$. The numerical part of a system of weights will be viewed up to 1 -coboundaries with values in $\mathbb{Z} / 2 \mathbb{Z}$, namely the numerical weights of all 4 edges incident to a vertex can simultaneously change by 1 . We next define the weighted fusion rules of Figure 39.

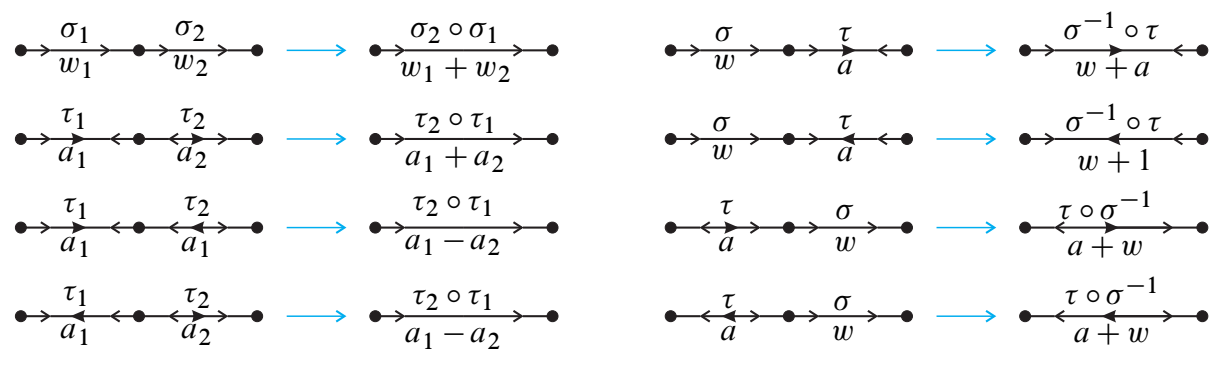

Figure 39: Edge fusion rules for graphs in $\widetilde{\mathcal{A}}_{\mathrm{w}}$

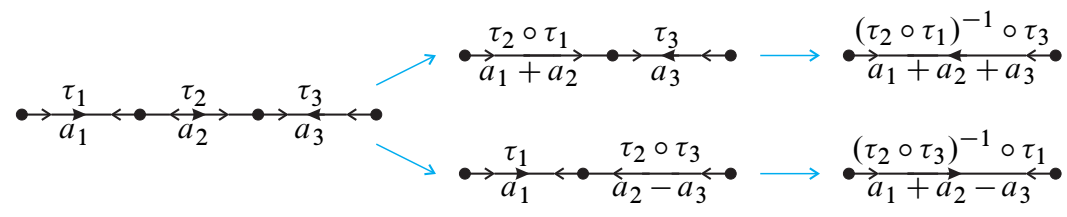

Figure 40: The fusion rules for graphs in $\widetilde{\mathcal{A}}_{\mathrm{w}}$ are not associative. In this example both the internal orientation and the numerical weight $\pm \frac{1}{2}$ depend on the order in which fusions are performed; note however that $\left(\tau_{2} \circ \tau_{1}\right)^{-1} \circ \tau_{3}=$ $\left(\tau_{2} \circ \tau_{3}\right)^{-1} \circ \tau_{1}$, coherently with the fact that the fusion rules for unweighted graphs are associative.

Remark 4.5 The fusion rules do not cover the case of two odd edges with internal orientations both opposite to the external orientation after fusion, because this case will never occur for us. For the fusion of two odd edges with discordant internal orientations, we note that $a_{1}, a_{2}$ are $\pm \frac{1}{2}$, so $a_{1}-a_{2}=a_{2}-a_{1}$ in $\mathbb{Z} / 2 \mathbb{Z}$. 
The following fact, proved in Figure 40, must be taken into account.

Proposition 4.6 The weighted fusion rules of Figure 39 are not associative.

\subsection{Moves on weighted graphs}

We now introduce certain moves on $\mathcal{N}_{\mathrm{w}}$, to define which we also use $\widetilde{\mathcal{A}}_{\mathrm{w}}$. To begin we call elementary move on $\widetilde{\mathcal{A}}_{\mathrm{w}}$ one of $I, \bar{I}, I I, \overline{I I}, M, \bar{M}$ from Figures 41 and 42 .

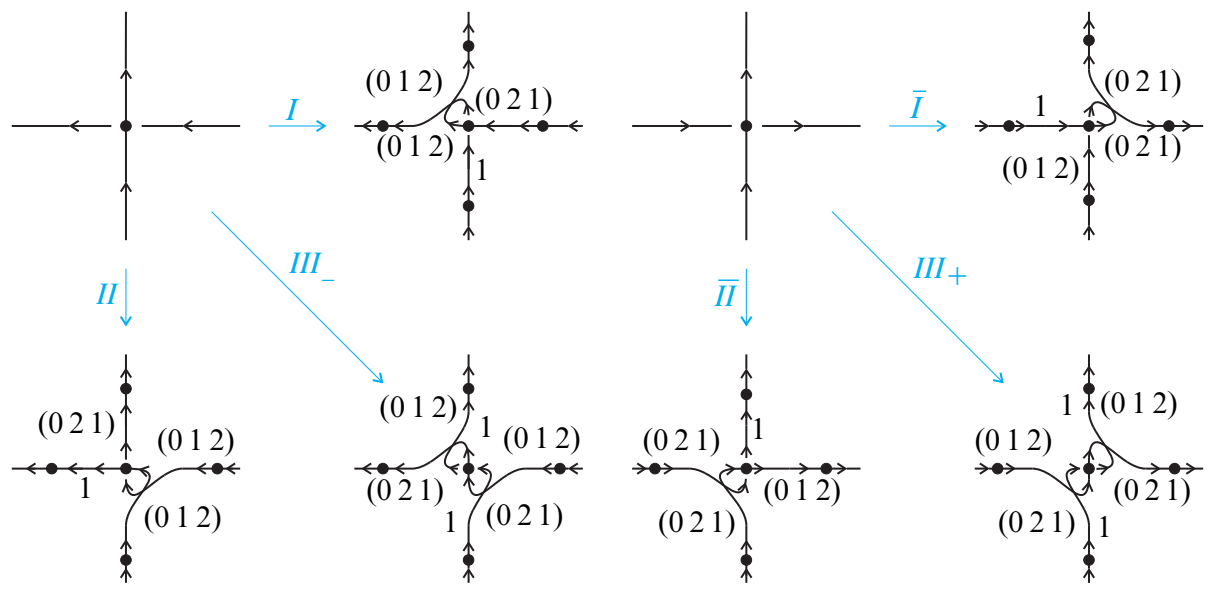

Figure 41: Moves on $\widetilde{\mathcal{A}}_{\mathrm{w}}$ derived from those on $\widetilde{\mathcal{N}}_{\mathrm{w}}$
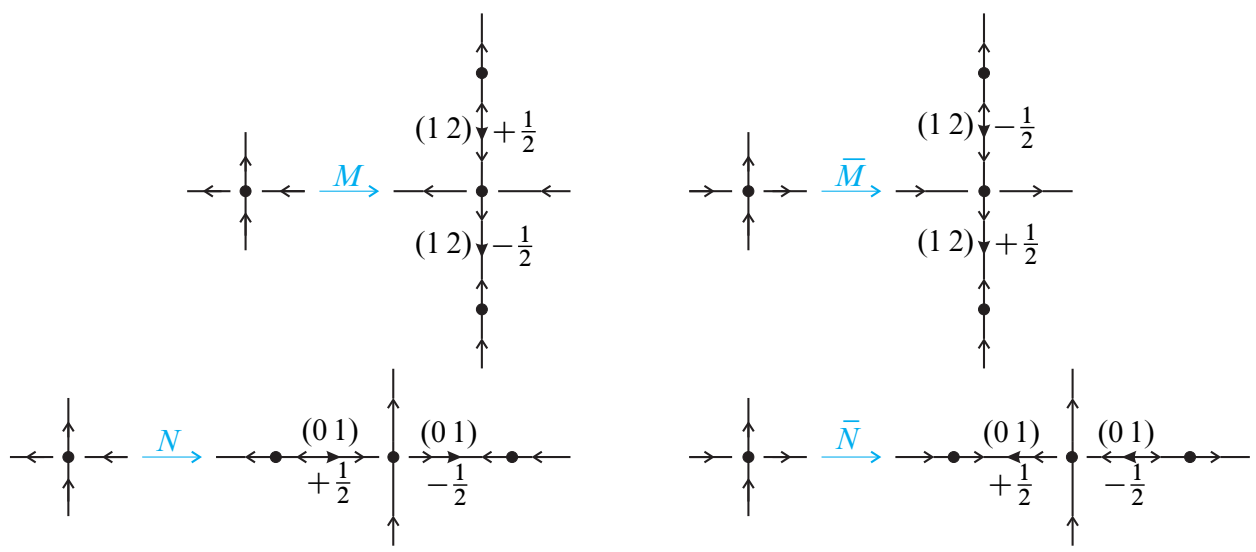

Figure 42: More moves on weighted graphs

The pictures contain more moves whose role will be explained soon. 
Remark 4.7 - In the symbols denoting the moves, overlining and subscripts are used to indicate the type of index transition $\pm 1 \mapsto \pm 1$.

- The moves $I$ and $I I$ are those of Figure 24 and at the level of $\mathcal{N}_{\mathrm{w}}$, namely under the associative fusion rules for $\widetilde{\mathcal{N}}_{\mathrm{W}}$, we have $\bar{I}=I^{-1}$ and $\overline{I I}=I I^{-1}$; moreover $I I I_{-}=I \cdot \bar{I}=I I \cdot \bar{I}$ and $I I I_{+}=\bar{I} \cdot I I=\bar{I} \cdot I$.

- In the product of two moves, the move to the left applies first; moreover, not all products make sense.

We now establish some results concerning relations between moves.

Proposition 4.8 Consider a vertex as in Figure 13, apply to it one of the following combination of weighted moves, and locally apply near the vertex the weighted fusion rules of Figure 39; then the result is the same as indicated:

$$
M \cdot \bar{M}=\mathrm{id}_{-}, \quad N \cdot \bar{N}=\mathrm{id}_{-}, \quad \bar{M} \cdot M=\mathrm{id}_{+}, \quad \bar{N} \cdot N=\mathrm{id}_{+}
$$

$$
I I I_{-} \cdot M=N \cdot I I I_{+}, \quad I I I_{+} \cdot \bar{M}=\bar{N} \cdot I I I_{-}, \quad M \cdot \bar{N}=N \cdot \bar{M}, \quad \bar{M} \cdot N=\bar{N} \cdot M
$$

Remark 4.9 Since the fusion rules in $\widetilde{\mathcal{A}}_{\mathrm{w}}$ are not associative, these equalities do not imply that at the level $\mathcal{A}_{\mathrm{w}}$ we have the relations

$$
\bar{M}=M^{-1}, \quad \bar{N}=N^{-1}, \quad N=I I I_{-} \cdot M \cdot I I I_{+},
$$

but these relations do make sense and hold in a restricted context; see below.

The proofs of some of the equalities in Proposition 4.8 are given in Figures 43-45; they all crucially use the weighted fusion rules of Figure 39 and the convention that weights are viewed up to $\mathbb{Z} / 2 \mathbb{Z}$-coboundaries; the other proofs are similar.

We now call weighted move on a vertex as in Figure 13 any sequence of elementary weighted moves (not followed by any fusion). We first have the following.

Proposition 4.10 Take $\Gamma \in \mathcal{N}_{\mathrm{w}}$ and apply to each of its vertices a weighted move to get $\widetilde{\Gamma} \in \widetilde{\mathcal{A}}$. Suppose that by applying (in some order) the weighted fusion rules of Figure 39 one gets $\Theta \in \mathcal{N}_{\mathrm{w}}$. Then the system of weights on $\Theta$ is well-defined independently of the order of application of the weighted fusion rules. 


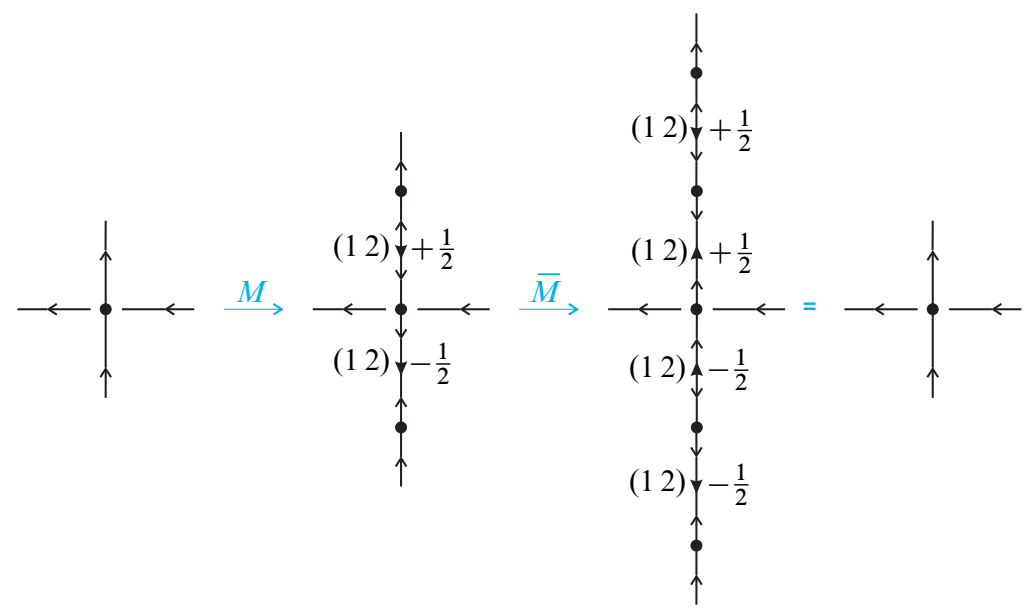

Figure 43: Proof that $M \cdot \bar{M}=\mathrm{id}_{-}$under local application of weighted fusion

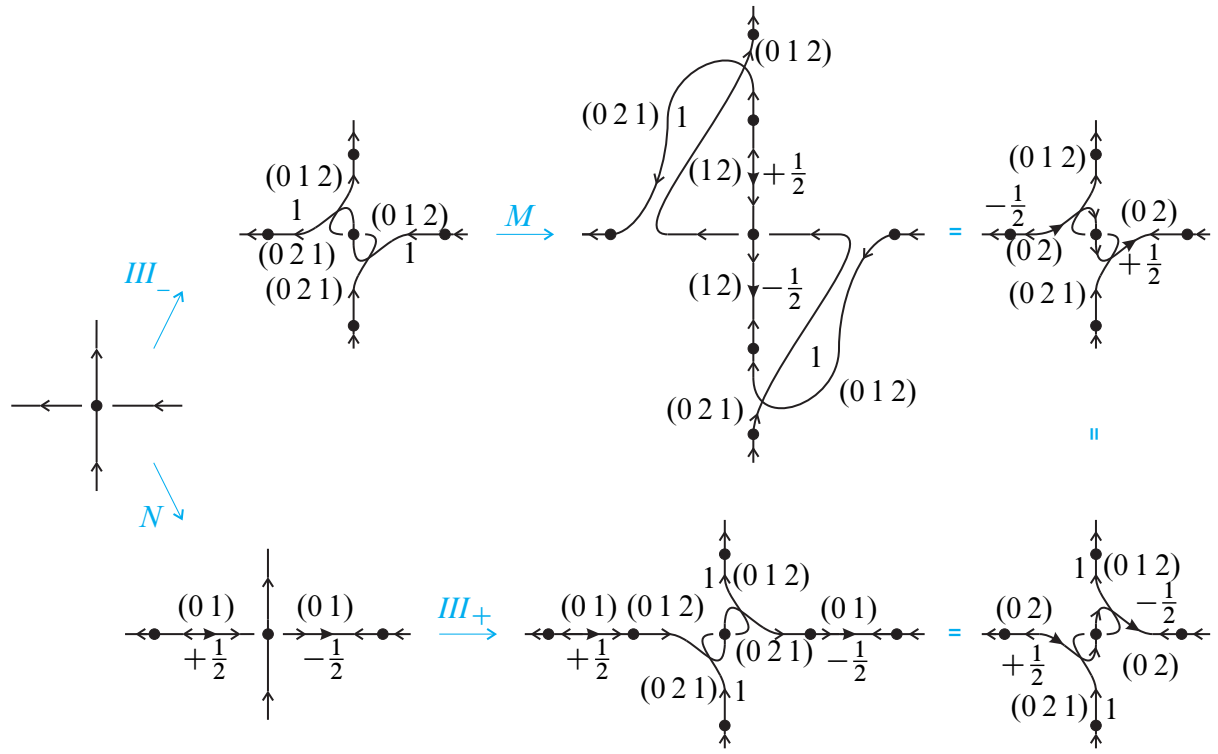

Figure 44: Proof that $I I I_{-} \cdot M=N \cdot I I I_{+}$under local application of weighted fusion

Proof The statement contains the implicit claim that the rules of Figure 39 suffice to go from $\widetilde{\Gamma}$ to some $\Theta$, namely that no situation as in Remark 4.5 occurs.

We prove the proposition ignoring the colors in $\mathfrak{S}_{3}$, because we already know that fusion is associative at the $\mathfrak{S}_{3}$ level. We concentrate on a single edge of $\Gamma$ and we imagine $e$ is initially drawn in front of us with orientation from left to right and 


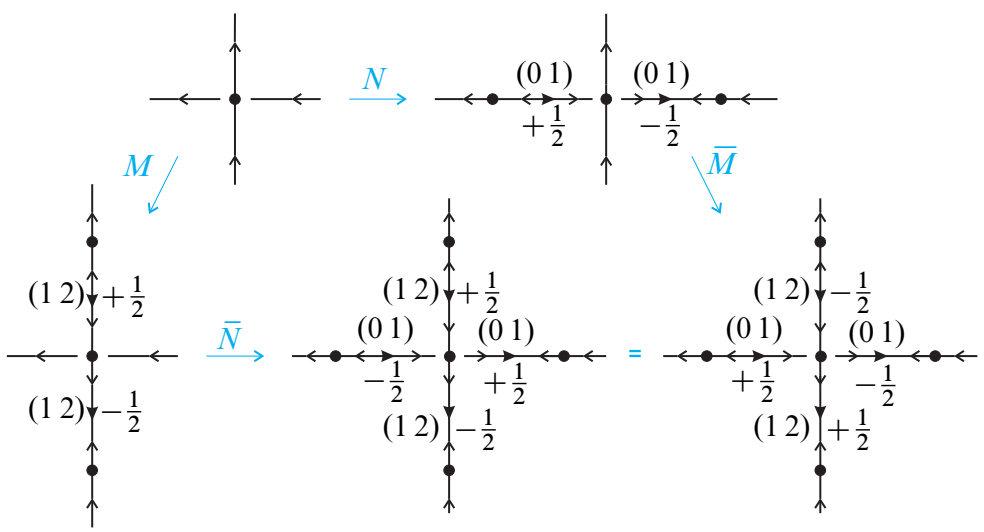

Figure 45: Proof that $M \cdot \bar{N}=N \cdot \bar{M}$ under local application of weighted fusion

weight $w \in\{0,1\}$. Replacing external orientations of edges by letters $r / \ell$ and internal orientations by $R / L$ we then have in $\Gamma$ an initial edge $\frac{r r}{w}$ that gets replaced in $\widetilde{\Gamma}$ by a concatenation $\tilde{e}$ of edges of the form

$$
\frac{r r}{u}, \quad \frac{\ell \ell}{u}, \quad \frac{r R \ell}{a}, \quad \frac{r L \ell}{a}, \quad \frac{\ell R r}{a}, \quad \frac{\ell L r}{a} .
$$

A careful examination of the elementary weighted moves actually shows that the possibilities for $\widetilde{e}$ are only as follows:

$$
\begin{gathered}
\left(\left(\frac{r r}{u}\right)^{*} \cdot \frac{r R \ell}{a} \cdot\left(\frac{\ell \ell}{u}\right)^{*} \cdot \frac{\ell L r}{a}\right)^{*} \cdot\left(\frac{r r}{u}\right)^{*} \cdot \frac{r r}{w} \cdot\left(\frac{r r}{u}\right)^{*} \cdot\left(\frac{r L \ell}{a} \cdot\left(\frac{\ell \ell}{u}\right)^{*} \cdot \frac{\ell R r}{a} \cdot\left(\frac{r r}{u}\right)^{*}\right)^{*} \\
\left(\frac{\ell \ell}{u}\right)^{*} \cdot \frac{\ell L r}{a} \cdot(4) \cdot \frac{r L \ell}{a} \cdot\left(\frac{\ell \ell}{u}\right)^{*}
\end{gathered}
$$

where $y^{*}$ means any number (including 0 ) of repetitions of a string $y$, and the weight $u$ (resp. a) can have a different value in $\{0,1\}$ (resp. $\pm \frac{1}{2}$ ) each time it appears. It is then clear that we never get any of the adjacencies $\frac{r L \ell}{a} \cdot \frac{\ell L r}{a}$ or $\frac{\ell R r}{a} \cdot \frac{r R \ell}{a}$ not contemplated by the weighted fusion rules of Figure 39. Moreover these rules can be expressed as

$$
\begin{gathered}
\frac{r r}{u_{1}} \cdot \frac{r r}{u_{2}}=\frac{r r}{u_{1}+u_{2}}, \quad \frac{r r}{u} \cdot \frac{r D \ell}{a}=\frac{r D \ell}{u+a}, \quad \frac{\ell D r}{a} \cdot \frac{r r}{u}=\frac{\ell D r}{a+u}, \\
\frac{\ell \ell}{u_{1}} \cdot \frac{\ell \ell}{u_{2}}=\frac{\ell \ell}{u_{1}+u_{2}}, \quad \frac{\ell \ell}{u} \cdot \frac{\ell D r}{a}=\frac{\ell D r}{u+a}, \quad \frac{r D \ell}{a} \cdot \frac{\ell \ell}{u}=\frac{r D \ell}{a+u}, \\
\frac{\ell L r}{a_{1}} \cdot \frac{r L \ell}{a_{2}}=\frac{\ell \ell}{a_{1}+a_{2}}, \quad \frac{r R \ell}{a_{1}} \cdot \frac{\ell R r}{a_{2}}=\frac{r r}{a_{1}+a_{2}}, \\
\frac{\ell L r}{a_{1}} \cdot \frac{r R \ell}{a_{2}}=\frac{\ell \ell}{a_{1}-a_{2}}, \quad \frac{\ell R r}{a_{1}} \cdot \frac{r L \ell}{a_{2}}=\frac{\ell \ell}{a_{1}-a_{2}}, \quad \frac{r R \ell}{a_{1}} \cdot \frac{\ell L r}{a_{2}}=\frac{r r}{a_{1}-a_{2}}, \quad \frac{r L \ell}{a_{1}} \cdot \frac{\ell R r}{a_{2}}=\frac{r r}{a_{1}-a_{2}} .
\end{gathered}
$$

We must show that by applying them as long as possible to (4) or (5) we get a welldefined result. Note first that each edge $\frac{\ell \ell}{u}$ or $\frac{r r}{u}$ can be ignored; in fact, its contribution is independent of the time it is involved in weighted fusions, because 
- on the internal orientation it acts identically to the right and to the left,

- its numerical weight is in $\{0,1\}$, so it is insensitive to later sign change.

We then have to deal with concatenations of the form

$$
\begin{gathered}
\frac{r R \ell}{a_{1}} \cdot \frac{\ell L r}{b_{1}} \ldots \frac{r R \ell}{a_{k}} \cdot \frac{\ell L r}{b_{k}} \cdot \frac{r L \ell}{d_{h}} \cdot \frac{\ell R r}{c_{h}} \ldots \frac{r L \ell}{d_{1}} \cdot \frac{\ell R r}{c_{1}}, \\
\frac{\ell L r}{b_{0}} \cdot \frac{r R \ell}{a_{1}} \cdot \frac{\ell L r}{b_{1}} \ldots \frac{r R \ell}{a_{k}} \cdot \frac{\ell L r}{b_{k}} \cdot \frac{r L \ell}{d_{h}} \cdot \frac{\ell R r}{c_{h}} \cdots \frac{r L \ell}{d_{1}} \cdot \frac{\ell R r}{c_{1}} \cdot \frac{r L \ell}{d_{0}},
\end{gathered}
$$

but we also consider the following (that arise starting from an edge $\frac{\ell \ell}{w}$ in $\Gamma$ ):

$$
\begin{gathered}
\frac{\ell L r}{a_{1}} \cdot \frac{r R \ell}{b_{1}} \ldots \frac{\ell L r}{a_{k}} \cdot \frac{r R \ell}{b_{k}} \cdot \frac{\ell R r}{d_{h}} \cdot \frac{r L \ell}{c_{h}} \ldots \frac{\ell R r}{d_{1}} \cdot \frac{r L \ell}{c_{1}} \\
\frac{r R \ell}{b_{0}} \cdot \frac{\ell L r}{a_{1}} \cdot \frac{r R \ell}{b_{1}} \ldots \frac{\ell L r}{a_{k}} \cdot \frac{r R \ell}{b_{k}} \cdot \frac{\ell R r}{d_{h}} \cdot \frac{r L \ell}{c_{h}} \ldots \frac{\ell R r}{d_{1}} \cdot \frac{r L \ell}{c_{1}} \cdot \frac{\ell R r}{d_{0}}
\end{gathered}
$$

We now claim that, regardless of the order in which the weighted fusion rules are applied, the numerical edge weight on the final result is

$$
\begin{gathered}
\sum_{i=1, \ldots, k} a_{i}-\sum_{i=1, \ldots, k} b_{i}+\sum_{j=1, \ldots, h} c_{j}-\sum_{j=1, \ldots, h} d_{j} \text { for (6) and (8), } \\
\sum_{i=1, \ldots, k} a_{i}-\sum_{i=0, \ldots, k} b_{i}+\sum_{j=1, \ldots, h} c_{j}-\sum_{j=0, \ldots, h} d_{j} \text { for (7) and (9). }
\end{gathered}
$$

The claim of course implies the conclusion, and we can prove it by induction on half the length of the concatenation. The base step of the induction is with length 0 in cases (6) and (8), so it is empty, and with length 2 in cases (7) and (9), so it follows directly from the weighted fusion rules (remember that $-b_{0}-d_{0}=b_{0}+d_{0}$ because both $b_{0}$ and $d_{0}$ are $\pm \frac{1}{2}$ ). For the inductive step we must analyze what happens by applying one weighted fusion to one of (6)-(9). In all four cases we can distinguish between the "central" fusion $\frac{\ell L r}{b_{k}} \cdot \frac{r L \ell}{d_{h}} \rightarrow \frac{\ell \ell}{b_{k}+d_{h}}$ or $\frac{r R \ell}{b_{k}} \cdot \frac{\ell R r}{d_{h}} \rightarrow \frac{r r}{b_{k}+d_{h}}$ and any "lateral" fusion. Dealing with lateral fusions is easier, and we make it explicit only for case (6) and for a fusion performed to the left of the centre; this fusion will be $\frac{r R \ell}{a_{t}} \cdot \frac{\ell L r}{b_{t}} \rightarrow \frac{r r}{a_{t}-b_{t}}$ or $\frac{\ell L r}{b_{t}} \cdot \frac{r R \ell}{a_{t+1}} \rightarrow \frac{\ell \ell}{b_{t}-a_{t+1}}=\frac{\ell \ell}{a_{t+1}-b_{t}}$; then we can forget the fused edge (remembering that its weight must be added to the final one) so we are led to a shorter concatenation of type (6); the inductive assumption then easily implies the conclusion.

Turning to the central fusion, in case (6) forgetting the fused edge we get the shorter concatenation of type (9),

$$
\frac{r R \ell}{a_{1}} \cdot \frac{\ell L r}{b_{1}} \cdot \frac{r R \ell}{a_{2}} \cdots \frac{\ell L r}{b_{k-1}} \cdot \frac{r R \ell}{a_{k}} \cdot \frac{\ell R r}{c_{h}} \cdot \frac{r L \ell}{d_{h-1}} \cdots \frac{\ell R r}{c_{2}} \cdot \frac{r L \ell}{d_{1}} \cdot \frac{\ell R r}{c_{1}},
$$


whence, by the inductive assumption, independently of the order, a final weight

$$
\text { (10) } \begin{aligned}
b_{k}+d_{h}+\sum_{i=1, \ldots, k-1} b_{i}- & \sum_{i=1, \ldots, k} a_{i}+\sum_{j=1, \ldots, h-1} d_{j}-\sum_{j=1, \ldots, h} c_{j} \\
& =\sum_{i=1, \ldots, k} a_{i}-\sum_{i=1, \ldots, k} b_{i}+\sum_{j=1, \ldots, h} c_{j}-\sum_{j=1, \ldots, h} d_{j}
\end{aligned}
$$

as desired. The central fusion in (7) gives the type (8) concatenation

$$
\frac{\ell L r}{b_{0}} \cdot \frac{r R \ell}{a_{1}} \cdots \frac{\ell L r}{b_{k-1}} \cdot \frac{r R \ell}{a_{k}} \cdot \frac{\ell R r}{c_{h}} \cdot \frac{r L \ell}{d_{h-1}} \cdots \frac{\ell R r}{c_{1}} \cdot \frac{r L \ell}{d_{0}},
$$

whence final weight precisely as in (10), as desired. The central fusion in cases (8) and (9) is reduced in a similar way to the inductive assumption in cases (7) and (6) respectively.

Corollary 4.11 If a sequence of elementary weighted moves is applied to a vertex as in Figure 13 and the weighted fusions are applied as long as possible to the edges generated by these moves, the result is independent of the order of fusions.

Proof By the argument showing Proposition 4.10 we must prove that concatenations of the form

$$
\begin{array}{cl}
\frac{r R \ell}{a_{1}} \cdot \frac{\ell L r}{b_{1}} \cdots \frac{r R \ell}{a_{k}} \cdot \frac{\ell L r}{b_{k}}, & \frac{\ell L r}{a_{1}} \cdot \frac{r R \ell}{b_{1}} \cdots \frac{\ell L r}{a_{k}} \cdot \frac{r R \ell}{b_{k}}, \\
\frac{\ell L r}{b_{0}} \cdot \frac{r R \ell}{a_{1}} \cdot \frac{\ell L r}{b_{1}} \cdots \frac{r R \ell}{a_{k}} \cdot \frac{\ell L r}{b_{k}}, & \frac{r R \ell}{b_{0}} \cdot \frac{\ell L r}{a_{1}} \cdot \frac{r R \ell}{b_{1}} \cdots \frac{\ell L r}{a_{k}} \cdot \frac{r R \ell}{b_{k}},
\end{array}
$$

give a well-defined result. By induction on the length one can indeed see that the first two give $\sum_{i=1, \ldots, k} b_{i}-\sum_{i=1, \ldots, k} a_{i}$ and the last two give $\sum_{i=0, \ldots, k} b_{i}-\sum_{i=1, \ldots, k} a_{i}$

The two previous results imply that

- we can define a weighted move at a vertex as in Figure 13 as a sequence of elementary weighted moves followed by weighted fusion;

- if we apply to a graph in $\mathcal{N}_{\mathrm{w}}$ some weighted moves and after weighted fusion we get another graph in $\mathcal{N}_{\mathrm{w}}$, the weights on this last graph are well-defined.

Proposition 4.12 Two weighted moves at a vertex that coincide as unweighted moves also coincide as weighted moves.

Proof We will prove the result for moves turning a vertex of index -1 to another vertex of index -1 , the general case following by precomposition with move $I$ and/or postcomposition with move $\bar{I}$. The 12 moves described form a group $\Pi_{-}$which is 
intrinsically isomorphic to the alternating group $\mathfrak{S}_{4}^{+}$on 4 objects. This isomorphism is made explicit with the choice of generators and the resulting presentation as follows:

$$
\alpha=I I I_{-}, \quad \beta=I \cdot \bar{M}, \quad \Pi_{-}=\left\langle\alpha, \beta \mid \alpha^{2}, \beta^{3},(\alpha \cdot \beta)^{3}\right\rangle
$$

(with moves and relations understood without weights). To conclude it is then sufficient to show that the three relations hold also in a weighted sense. For $\alpha^{2}$ this was already implicit above and very easy anyway; the other two weighted relations are established in Figures 46 and 47.
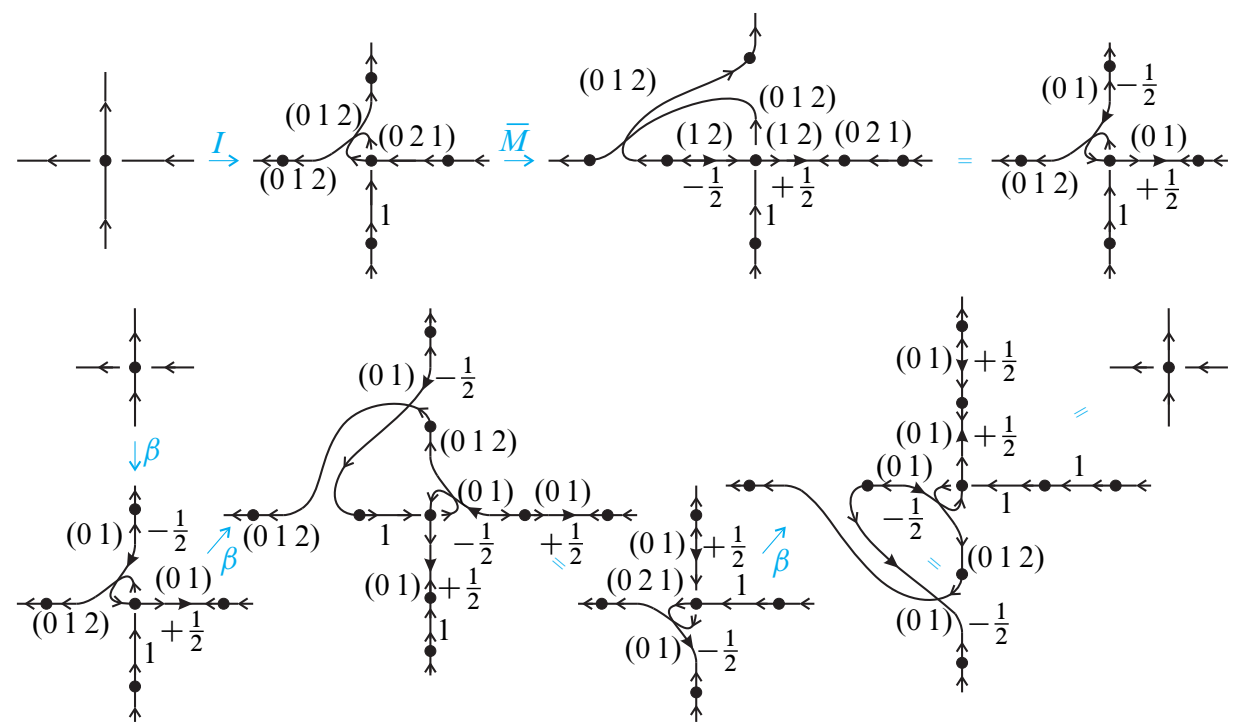

Figure 46: Computation of $\beta=I \cdot \bar{M}$ and proof that $\beta^{3}=\mathrm{id}_{-}$in a weighted sense

We are ready to establish our main result of this section.

Theorem 4.13 Two graphs in $\mathcal{N}_{\mathrm{w}}$ represent the same spine of some manifold $M$ and the same spin structure $s$ on $M$ if and only if they can be obtained from each other by a combination of the moves $I, I I, M, \bar{I}, \overline{I I}, \bar{M}$ and weighted fusion.

Proof Suppose that $\Gamma_{1}, \Gamma_{2} \in \mathcal{N}_{\mathrm{w}}$ represent the same $(M, s)$. Then they are related by moves $I, I I, \bar{I}, \overline{I I}$ and circuit moves. To get the desired conclusion it is then enough to show that the moves $M, \bar{M}$ generate the circuit move, which is done in Figure 48 for an edge of a circuit with first end of index -1 (the cases with first end +1 are similar).

For the opposite implication we need two preliminary results. The proof of the first one is an easy variation of the argument showing Proposition 3.3. 


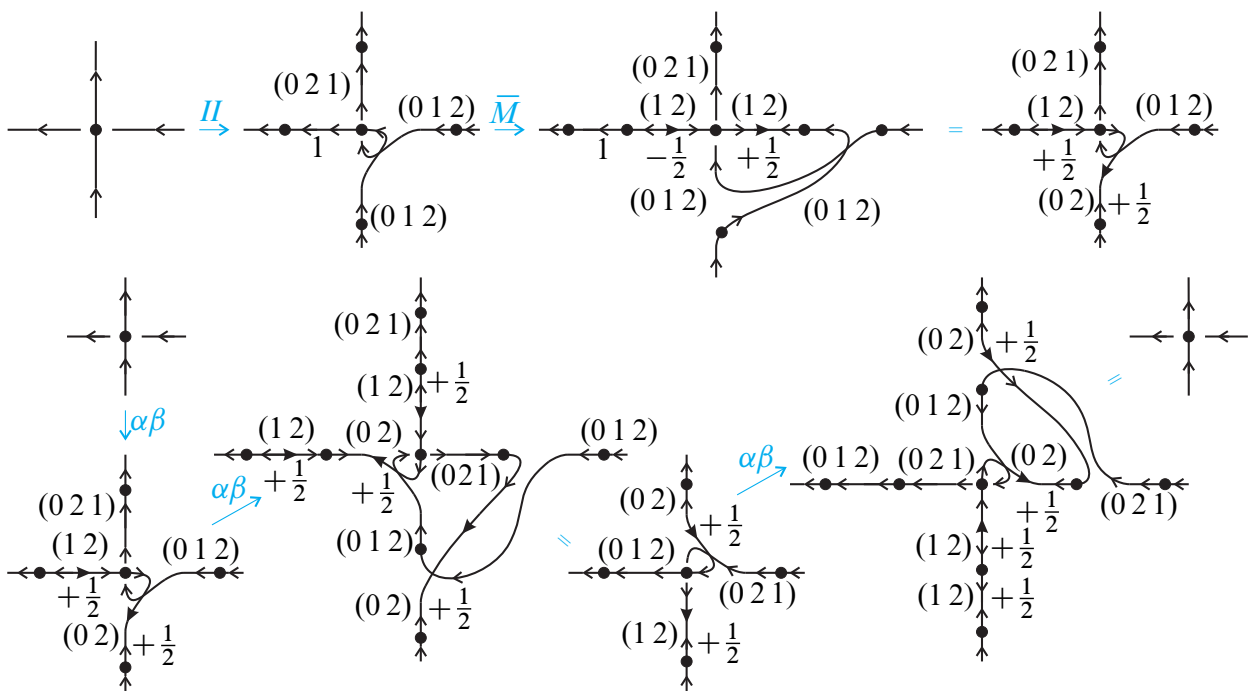

Figure 47: Computation of $\alpha \cdot \beta=I I I_{-} \cdot I \cdot \bar{M}=I I \cdot \bar{I} \cdot I \cdot \bar{M}=I I \cdot \bar{M}$ and proof that $(\alpha \cdot \beta)^{3}=$ id $_{-}$in a weighted sense
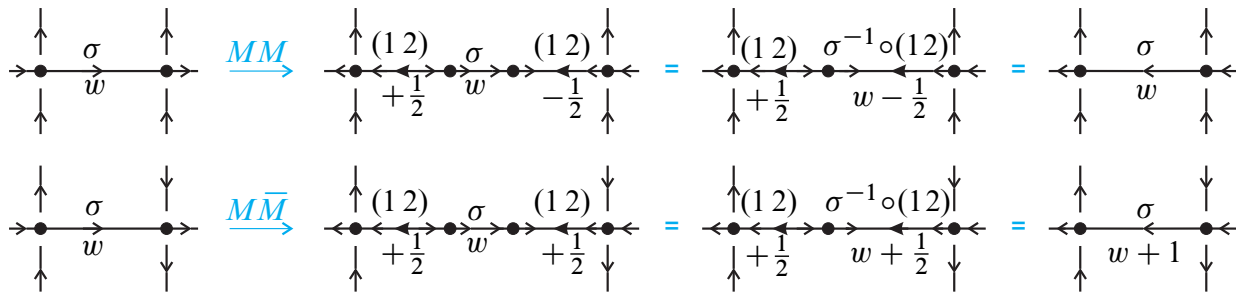

Figure 48: Generation of the circuit move via the moves $M, \bar{M}$ and weighted fusion

Proposition 4.14 Suppose that in $\Gamma \in \mathcal{N}_{\mathrm{w}}$ there are some (possibly intersecting) oriented circuits $\gamma_{1}, \ldots, \gamma_{n}$, and that each $\gamma_{j}$ is either an undercircuit (an underpass at all its vertices) or an overcircuit (an overpass at all its vertices). Then the spin structure defined by $\Gamma$ is also defined by the graph obtained from $\Gamma$ by reversing the orientation of each edge $e$ of $\gamma_{1} \cup \cdots \cup \gamma_{n}$ and adding 1 to the weight of $e$ if the ends if $e$ have different indices.

Proposition 4.15 If $\Gamma \in \mathcal{N}_{\mathrm{w}}$ then using the moves $I, I I, \bar{I}, \overline{I I}$ at the vertices of $\Gamma$, followed by fusion, one can get $\Theta \in \mathcal{N}_{\mathrm{w}}$ such that each edge of $\Theta$ is either an overpass at both its ends or an underpass at both its ends.

Proof To begin we note that given a vertex $V$ of $\Gamma$ and the choice of two germs of edges of $\Gamma$ at $V$ having consistent orientation through $V$, the moves $I, I I, \bar{I}, \overline{I I}$ allow 
to put the two chosen germs of edges in the overpass position at $v$. It is then enough to show that we can attach labels $o$ (over) and $u$ (under) to the germs of edges of $\Gamma$ at vertices, so that

- for each edge the labels at its ends are the same,

- at each vertex the germs having the same label have consistent orientation.

One such labeling will be termed good, and the coming argument proving its existence is due to Federico Petronio. We choose a vertex $V$ of $\Gamma$ and attach any label to any of the germs of edge at $V$. Then we propagate the labeling along a path in $\Gamma$ by applying alternatively the following rules until $V$ is reached again:

- if an end of an edge has a label, give the other end the same label;

- if at a vertex an incoming (respectively, outgoing) germ has a label, give the other incoming (respectively, outgoing) germ the other label.

Note that the propagation path need not be simple, but at each vertex visited twice the labeling is good; see Figure 49(left). When $V$ is reached again we have one of
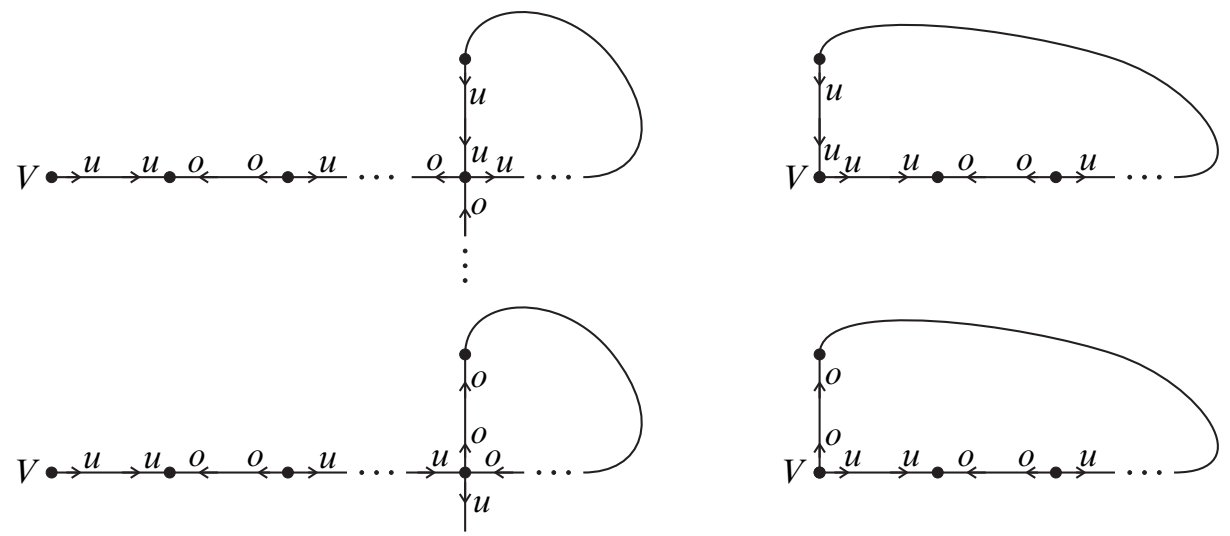

Figure 49: Extension of the labeling in case of initial label $u$ on an outgoing germ at $V$

the situations in Figure 49(right); in the top one we proceed by applying the second rule, and eventually get back to $V$ again with a good labeling; in the bottom one we proceed with an arbitrary choice of the label, but once more we get back to $V$ with a good labeling. We can now similarly start from some other vertex, until all the germs of edges at vertices are labeled. 
Back to the proof of Theorem 4.13, suppose that $\Gamma_{2} \in \mathcal{N}_{\mathrm{w}}$ is obtained from $\Gamma_{1} \in \mathcal{N}_{\mathrm{w}}$ by a combination of weighted moves $I, I I, M, \bar{I}, \overline{I I}, \bar{M}$ and weighted fusion. Let $\Delta$ be the union of the edges of $\Gamma_{2}$ having a different orientation in $\Gamma_{1}$. By Proposition 4.15 we can find weighted moves generated by $I, I I$ turning $\Gamma_{2}$ into $\Gamma_{3} \in \mathcal{N}_{\mathrm{w}}$ in which $\Delta$ appears as a union of overcircuits and undercircuits. Note that $\Gamma_{3}$ carries the same spin structure as $\Gamma_{2}$ by Proposition 3.1. With pictures similar to Figure 48 one can now see that the multiple circuit moves of Proposition 4.14 are generated by the moves $M, \bar{M}, N, \bar{N}, D_{-}=M \cdot \bar{N}=N \cdot \bar{M}, D_{+}=\bar{M} \cdot N=\bar{N} \cdot M$ (the move $D_{-}$is shown in Figure 45 , and $D_{+}$is obtained similarly).

This shows that we can find a combination of the moves $I, I I, M, \bar{I}, \overline{I I}, \bar{M}$ that, after weighted fusion, turn $\Gamma_{2}$ into some $\Gamma_{4}$ carrying the same spin structure as $\Gamma_{2}$ and the same prebranching as $\Gamma_{1}$. Proposition 3.1 then implies that via moves $I, I I, \bar{I}, \overline{I I}$ we can turn $\Gamma_{4}$ into some $\widetilde{\Gamma}_{1}$ carrying the same spin structure as $\Gamma_{2}$ and different from $\Gamma_{1}$ possibly only for the weights. We then have a sequence of weighted moves $I, I I, M, \bar{I}, \bar{I}, \bar{M}$ that under weighted fusion give

$$
\Gamma_{1} \longrightarrow \Gamma_{2} \longrightarrow \Gamma_{3} \longrightarrow \Gamma_{4} \longrightarrow \widetilde{\Gamma}_{1}
$$

and that ignoring weights give the identity of $\Gamma_{1}$ (namely, they give the identity at every vertex of $\Gamma_{1}$ ). Proposition 4.12 then implies that $\widetilde{\Gamma}_{1}$ coincides with $\Gamma_{1}$ also as a weighted graph (up to coboundaries). This shows that $\Gamma_{2}$ carries the same spin structure as $\Gamma_{1}$.

\subsection{Obstruction computation on graphs with split edges}

Even if this is not strictly necessary for our main results, we provide here two methods for the computation of the obstruction $\alpha(P, \omega, b)$ carried by a graph $\widetilde{\Gamma} \in \widetilde{\mathcal{A}}$ that after fusion becomes a graph in $\Theta \in \mathcal{N}$ defining a triple $(P, \omega, b)$. The first method is general, direct and easy; the second one only applies to a $\widetilde{\Gamma}$ resulting from the application to some $\Gamma \in \mathcal{N}$ of the moves of Proposition 4.10 (ignoring the numerical weights but using internal orientations), and it is more complicated, but it also shows that some nontrivial algebra underlies the computation.

First method Take $\tilde{\Gamma} \in \tilde{\mathcal{A}}$ that after fusion gives $\Gamma \in \mathcal{N}$ representing $(P, \omega, b)$. We claim that $\alpha(P, \omega, b)$ can be computed from $\widetilde{\Gamma}$ by considering on the boundary of each region of $P$ some numerical contributions in $G=\left(\frac{1}{2} \cdot \mathbb{Z}\right) / 2 \mathbb{Z}$ and some arrows, as in Proposition 2.7. Contributions from vertices and from even edges are the same as in Proposition 2.7, while those from an odd edge $e$ are described as follows (with the regions labeled 0,1,2 as in Figure 33 and contributions 0 not mentioned): 


\begin{tabular}{c||c|c|c}
$e$ & $\tau=\left(\begin{array}{ll}0 & 1\end{array}\right)$ & $\tau=\left(\begin{array}{ll}0 & 2\end{array}\right)$ & $\tau=\left(\begin{array}{ll}1 & 2\end{array}\right)$ \\
\hline \hline$\stackrel{\tau}{\longrightarrow} \bullet$ & regions 0 and 1 get $+\frac{1}{2}$ & all regions get 1 & regions 1 and 2 get $-\frac{1}{2}$ \\
\hline$\stackrel{\tau}{\longrightarrow} \cdot$ & regions 0 and 1 get $-\frac{1}{2}$ & all regions get 1 & regions 1 and 2 get $+\frac{1}{2}$
\end{tabular}

The proof that this recipe works follows from the fact that the contributions combine consistently under fusion, which is shown on examples in Figure 50.
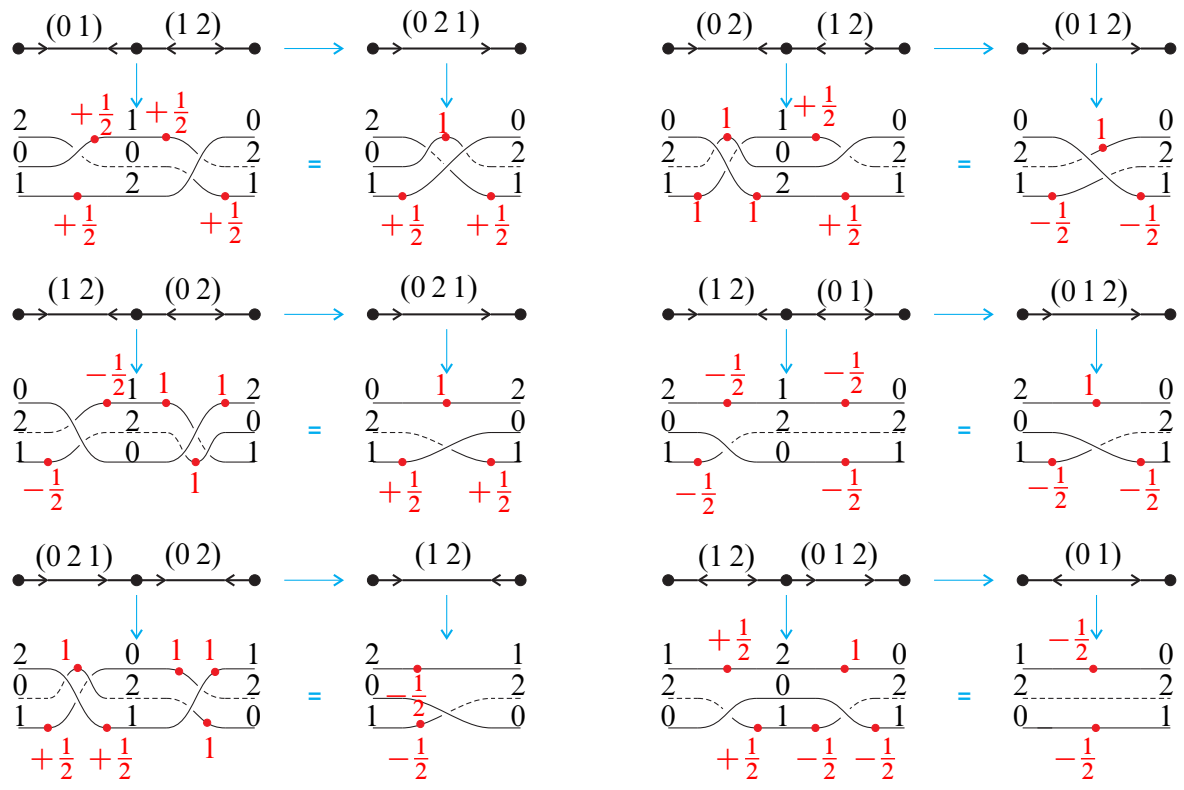

Figure 50: Associativity of the computation of $\alpha$ on a graph in $\widetilde{\mathcal{A}}$

Second method We begin with an apparently unrelated algebraic result. For any set $G$ we consider the right action of $\mathfrak{S}_{3}$ on $G$ given by

$$
\left(g_{0}, g_{1}, g_{2}\right) \cdot \eta=\left(g_{\eta(0)}, g_{\eta(1)}, g_{\eta(2)}\right) .
$$

We check that indeed this is a right action on an example:

$$
\begin{aligned}
& \left(\left(g_{0}, g_{1}, g_{2}\right) \cdot(01)\right) \cdot(12)=\left(g_{1}, g_{0}, g_{2}\right) \cdot(12)=\left(g_{1}, g_{2}, g_{0}\right) \\
& \left(g_{0}, g_{1}, g_{2}\right) \cdot\left(\left(\begin{array}{lll}
0 & 1
\end{array}\right) \circ\left(\begin{array}{ll}
1 & 2
\end{array}\right)\right)=\left(g_{0}, g_{1}, g_{2}\right) \cdot\left(\begin{array}{lll}
0 & 1 & 2
\end{array}\right)=\left(g_{1}, g_{2}, g_{0}\right)
\end{aligned}
$$

If $G$ is an abelian group of course we have

$$
\left(\left(g_{0}, g_{1}, g_{2}\right)+\left(h_{0}, h_{1}, h_{2}\right)\right) \cdot \eta=\left(g_{0}, g_{1}, g_{2}\right) \cdot \eta+\left(h_{0}, h_{1}, h_{2}\right) \cdot \eta
$$


so we can define the semidirect product $\mathfrak{S}_{3} \amalg G^{3}$ as $\mathfrak{S}_{3} \times G^{3}$ with operation

$$
\left(\eta,\left(g_{0}, g_{1}, g_{2}\right)\right) \cdot\left(\theta,\left(h_{0}, h_{1}, h_{2}\right)\right)=\left(\eta \circ \theta,\left(g_{0}, g_{1}, g_{2}\right) \cdot \theta+\left(h_{0}, h_{1}, h_{2}\right) .\right.
$$

We now specialize our choice to $=\left(\frac{1}{2} \cdot \mathbb{Z}\right) / 2 \mathbb{Z}$ and we establish the following.

Proposition 4.16 Define $s: \mathfrak{S}_{3} \rightarrow G^{3}$ by

$$
\begin{aligned}
& s(\emptyset)=(0,0,0), \quad s\left(\left(\begin{array}{lll}
0 & 1 & 2
\end{array}\right)\right)=\left(-\frac{1}{2},-\frac{1}{2}, 1\right), \quad s\left(\left(\begin{array}{lll}
0 & 1
\end{array}\right)\right)=\left(-\frac{1}{2},+\frac{1}{2}, 0\right), \\
& s((02))=(1,0,1), \quad s\left(\left(\begin{array}{lll}
0 & 1
\end{array}\right)\right)=\left(1,+\frac{1}{2},+\frac{1}{2}\right), \quad s\left(\left(\begin{array}{ll}
1 & 2)
\end{array}\right)=\left(0,-\frac{1}{2},+\frac{1}{2}\right) .\right.
\end{aligned}
$$

Then $\Psi: \mathfrak{S}_{3} \rightarrow \mathfrak{S}_{3} \amalg G^{3}$ given by $\Psi(\eta)=(\eta, s(\eta))$ is a group homomorphism.

Proof If $x=\left(\begin{array}{ll}0 & 1)\end{array}\right)$ and $y=(12)$ we have the presentation of $\mathfrak{S}_{3}$ given by

$$
\left\langle x, y \mid x^{2}, y^{2},(x \cdot y)^{3}\right\rangle
$$

with $\left(\begin{array}{lll}0 & 1 & 2\end{array}\right)=x \cdot y,\left(\begin{array}{lll}0 & 2 & 1\end{array}\right)=y \cdot x,\left(\begin{array}{l}0 \\ 2\end{array}\right)=x \cdot y \cdot x$. The proposition will then be a consequence of the relations

$$
\begin{aligned}
& \Psi(x)^{2}=\Psi(y)^{2}=(\Psi(x) \cdot \Psi(y))^{3}=(\emptyset,(0,0,0)), \\
& \Psi\left(\left(\begin{array}{ll}
0 & 2))
\end{array}\right) \Psi(x) \cdot \Psi(y) \cdot \Psi(x),\right. \\
& \Psi\left(\left(\begin{array}{lll}
0 & 1 & 2
\end{array}\right)\right)=\Psi(x) \cdot \Psi(y), \\
& \Psi\left(\left(\begin{array}{lll}
0 & 2 & 1
\end{array}\right)\right)=\Psi(y) \cdot \Psi(x) .
\end{aligned}
$$

We start with

$$
\begin{aligned}
\Psi(x)^{2} & =\left((01),\left(-\frac{1}{2},+\frac{1}{2}, 0\right)\right) \cdot\left((01),\left(-\frac{1}{2},+\frac{1}{2}, 0\right)\right) \\
& =\left((01) \circ(01),\left(+\frac{1}{2},-\frac{1}{2}, 0\right)+\left(-\frac{1}{2},+\frac{1}{2}, 0\right)\right)=(\emptyset,(0,0,0)) .
\end{aligned}
$$

The computation of $\Psi(y)^{2}$ is similar. Before checking that $\Psi(x) \cdot \Psi(y)$ has vanishing

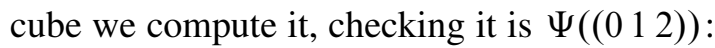

$$
\begin{aligned}
\Psi(x) \cdot \Psi(y) & =\left((01),\left(-\frac{1}{2},+\frac{1}{2}, 0\right)\right) \cdot\left((12)\left(0,-\frac{1}{2},+\frac{1}{2}\right)\right) \\
& =\left(\left(\begin{array}{lll}
0 & 1) \circ( & 1
\end{array}\right),\left(-\frac{1}{2}, 0,+\frac{1}{2}\right)+\left(0,-\frac{1}{2},+\frac{1}{2}\right)\right) \\
& =\left(\left(\begin{array}{lll}
0 & 1 & 2
\end{array}\right),\left(-\frac{1}{2},-\frac{1}{2}, 1\right)\right)
\end{aligned}
$$


And now we conclude

$$
\begin{aligned}
& \left(\Psi\left(\left(\begin{array}{lll}
0 & 1 & 2
\end{array}\right)\right)\right)^{3}=\left(\left(\begin{array}{lll}
0 & 1 & 2
\end{array}\right),\left(-\frac{1}{2},-\frac{1}{2}, 1\right)\right)^{3} \\
& =\left(\left(\begin{array}{lll}
0 & 1 & 2
\end{array}\right) \circ\left(\begin{array}{lll}
0 & 1 & 2
\end{array}\right),\left(-\frac{1}{2}, 1,-\frac{1}{2}\right)+\left(-\frac{1}{2},-\frac{1}{2}, 1\right)\right) \cdot \Psi\left(\left(\begin{array}{lll}
0 & 1 & 2
\end{array}\right)\right) \\
& =\left(\left(\begin{array}{lll}
0 & 2 & 1
\end{array}\right),\left(1,+\frac{1}{2},+\frac{1}{2}\right)\right) \cdot\left(\left(\begin{array}{lll}
0 & 1 & 2
\end{array}\right),\left(-\frac{1}{2},-\frac{1}{2}, 1\right)\right) \\
& =\left(\left(\begin{array}{lll}
0 & 2 & 1
\end{array}\right) \circ\left(\begin{array}{lll}
0 & 1 & 2
\end{array}\right),\left(+\frac{1}{2},+\frac{1}{2}, 1\right)+\left(-\frac{1}{2},-\frac{1}{2}, 1\right)\right)=(\emptyset,(0,0,0)) \text {, } \\
& \Psi(y) \cdot \Psi(x)=\left((12),\left(0,-\frac{1}{2},+\frac{1}{2}\right)\right) \cdot\left((01),\left(-\frac{1}{2},+\frac{1}{2}, 0\right)\right) \\
& =\left((12) \circ(01),\left(-\frac{1}{2}, 0,+\frac{1}{2}\right)+\left(-\frac{1}{2},+\frac{1}{2}, 0\right)\right) \\
& =\left(\left(\begin{array}{lll}
0 & 1 & 1
\end{array}\right),\left(1,+\frac{1}{2},+\frac{1}{2}\right)\right) \text {, } \\
& \Psi(x) \cdot \Psi(y) \cdot \Psi(x)=\left((01),\left(-\frac{1}{2},+\frac{1}{2}, 0\right)\right) \cdot\left((021),\left(1,+\frac{1}{2},+\frac{1}{2}\right)\right) \\
& =\left(\left(\begin{array}{lll}
0 & 1)
\end{array} \circ\left(\begin{array}{lll}
0 & 2 & 1
\end{array}\right),\left(0,-\frac{1}{2},+\frac{1}{2}\right)+\left(1,+\frac{1}{2},+\frac{1}{2}\right)\right)\right. \\
& =((02),(1,0,1)) \text {, }
\end{aligned}
$$

thus completing the proof.

Remark 4.17 The previous result remains true, with the same proof, if the values on $s$ on the transpositions are redefined as

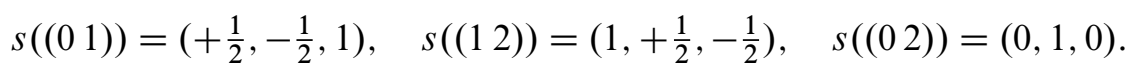

Let us then turn to the computation of the obstruction $\alpha(P, \omega, b)$. We start from $\Gamma \in \mathcal{N}$, we apply to it some of the moves of Proposition 4.10 (but neglecting the numerical weight) and we call $\widetilde{\Gamma}$ the result. Next, we assume that applying fusion to $\widetilde{\Gamma}$ we get $\Theta \in \mathcal{N}$ defining $(P, \omega, b)$. Note that every edge of $\widetilde{\Gamma}$ carries an internal orientation (that for an even edge we stipulate to be the same as the orientations at the ends). Let us concentrate on an edge $e$ of $\Theta$, that in $\widetilde{\Gamma}$ (before fusion) will be subdivided into several edges. Since in $\Theta \in \mathcal{N}$ the edge $e$ is oriented, we can speak of a global orientation of $e$ (that coincides with the internal orientations of the two extremal subedges of $e$ ). Now note that each subedge $e^{\prime}$ of $e$ brings three portions of strands of attaching circles of $P$ to $S(P)$, and that these strands are numbered $0,1,2$ at both ends of $e^{\prime}$ according to the orientation of these. The recipe for the computation of $\alpha(P, \omega, b)$ now uses the map $s$ of Proposition 4.16, and goes as follows:

- let $\eta \in \mathfrak{S}_{3}$ be the permutation attached to $e^{\prime}$, and define $\left(h_{0}, h_{1}, h_{2}\right)$ to be $s(\eta)$ if the internal orientation of $e^{\prime}$ is consistent with the global one, otherwise define $\left(h_{0}, h_{1}, h_{2}\right)$ as $s\left(\eta^{-1}\right)$;

- at the first end of $e^{\prime}$ with respect to the global orientation, attach to the strands $0,1,2$ the weights $h_{0}, h_{1}, h_{2}$. 
A formal proof that summing the contributions of the various $e^{\prime}$ one gets the edge contributions to $\alpha(P, \omega, b)$ as in Proposition 2.7 employs Proposition 4.16, but we confine ourselves here to some examples only; see Figures 51-56.

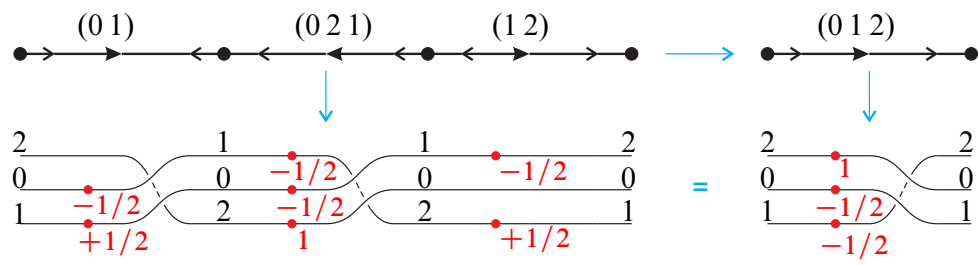

Figure 51: Computation of $\alpha$ with the second method, example 1

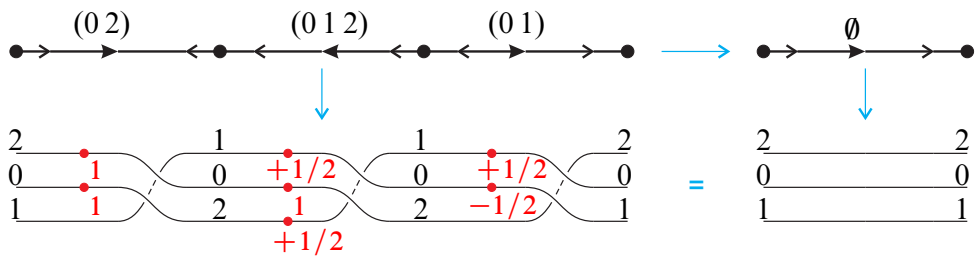

Figure 52: Computation of $\alpha$ with the second method, example 2

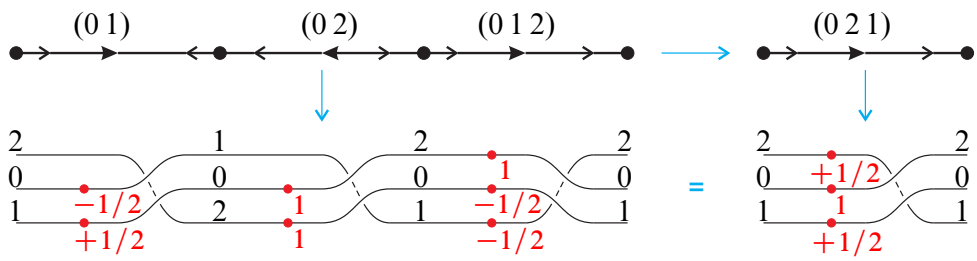

Figure 53: Computation of $\alpha$ with the second method, example 3

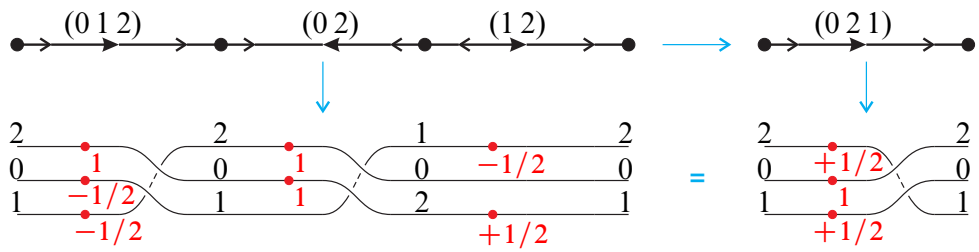

Figure 54: Computation of $\alpha$ with the second method, example 4 


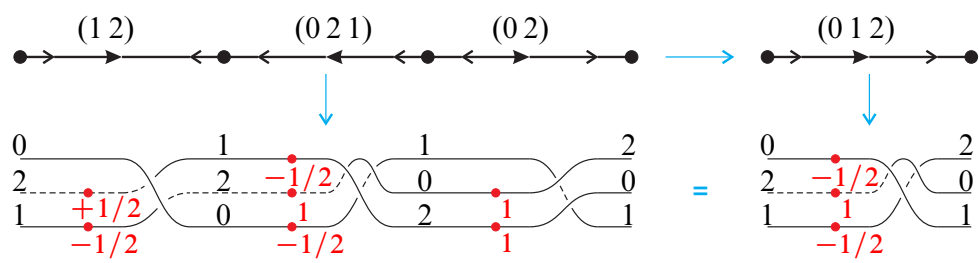

Figure 55: Computation of $\alpha$ with the second method, example 5
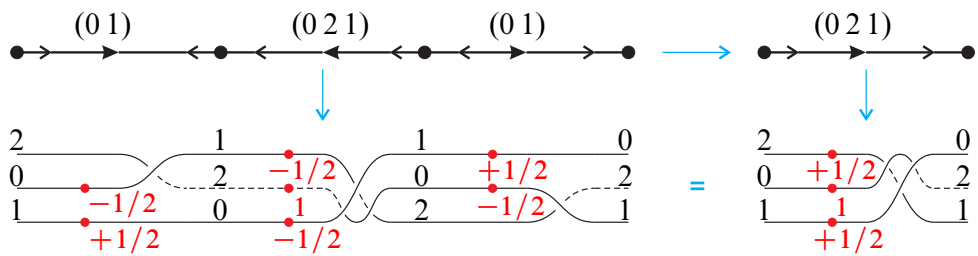

Figure 56: Computation of $\alpha$ with the second method, example 6

\section{References}

[1] G Amendola, R Benedetti, F Costantino, C Petronio, Branched spines of 3manifolds and Reidemeister torsion of Euler structures, Rend. Istit. Mat. Univ. Trieste 32 (2001) 1-33 MR1893390

[2] S Baseilhac, R Benedetti, Analytic families of quantum hyperbolic invariants and their asymptotical behaviour, I arXiv:1212.4261

[3] S Baseilhac, R Benedetti, Quantum hyperbolic invariants of 3-manifolds with PSL(2, C)-characters, Topology 43 (2004) 1373-1423 MR2081430

[4] S Baseilhac, R Benedetti, Classical and quantum dilogarithmic invariants of flat PSL(2, C)-bundles over 3-manifolds, Geom. Topol. 9 (2005) 493-569 MR2140989

[5] R Benedetti, C Petronio, A finite graphic calculus for 3-manifolds, Manuscripta Math. 88 (1995) 291-310 MR1359699

[6] R Benedetti, C Petronio, Branched standard spines of 3-manifolds, Lecture Notes in Mathematics 1653, Springer, Berlin (1997) MR1470454

[7] R Benedetti, C Petronio, Reidemeister-Turaev torsion of 3-dimensional Euler structures with simple boundary tangency and pseudo-Legendrian knots, Manuscripta Math. 106 (2001) 13-61 MR1860979

[8] C Blanchet, Invariants on three-manifolds with spin structure, Comment. Math. Helv. 67 (1992) 406-427 MR1171303

[9] J Christy, Branched surfaces and attractors, I: Dynamic branched surfaces, Trans. Amer. Math. Soc. 336 (1993) 759-784 MR1148043

[10] A Hatcher, Algebraic topology, Cambridge Univ. Press (2002) MR1867354 
[11] I Ishii, Flows and spines, Tokyo J. Math. 9 (1986) 505-525 MR875203

[12] F Luo, private communication (2011-2013)

[13] F Luo, J-M Schlenker, Volume maximization and the extended hyperbolic space, Proc. Amer. Math. Soc. 140 (2012) 1053-1068 MR2869090

[14] F Luo, S Tillmann, T Yang, Thurston's spinning construction and solutions to the hyperbolic gluing equations for closed hyperbolic 3-manifolds, Proc. Amer. Math. Soc. 141 (2013) 335-350 MR2988735

[15] S V Matveev, Transformations of special spines, and the Zeeman conjecture, Izv. Akad. Nauk SSSR Ser. Mat. 51 (1987) 1104-1116, 1119 MR925096 In Russian; translated in Math. USSR-Izv. 31 (1988) 423-434

[16] S V Matveev, Complexity theory of three-dimensional manifolds, Acta Appl. Math. 19 (1990) 101-130 MR1074221

[17] R Piergallini, Standard moves for standard polyhedra and spines, Rend. Circ. Mat. Palermo Suppl. (1988) 391-414 MR958750

[18] N Reshetikhin, V G Turaev, Invariants of 3-manifolds via link polynomials and quantum groups, Invent. Math. 103 (1991) 547-597 MR1091619

[19] J Roberts, Refined state-sum invariants of 3-and 4-manifolds, from: "Geometric topology", (W H Kazez, editor), AMS/IP Stud. Adv. Math. 2, Amer. Math. Soc. (1997) 217-234 MR1470729

[20] V G Turaev, O Y Viro, State sum invariants of 3-manifolds and quantum 6j-symbols, Topology 31 (1992) 865-902 MR1191386

[21] R F Williams, Expanding attractors, Inst. Hautes Études Sci. Publ. Math. (1974) 169203 MR0348794

Dipartimento di Matematica, Università di Pisa

Largo Bruno Pontecorvo 5, I-56127 Pisa, Italy

benedett@dm.unipi.it, petronio@dm.unipi.it

http://www.dm.unipi.it/ benedett/,

http://www.dm.unipi.it/pages/petronio/public_html/

Received: 16 April 2013 Revised: 9 September 2013 